\title{
Von Agenten, Akteuren und Strukturen in den Internationalen Beziehungen
}

\author{
Konturen einer empirischen Agency-Forschung
}

\begin{abstract}
Die Literatur der Internationalen Beziehungen hat sich ausgiebig mit dem AgentStruktur-Dilemma beschäftigt. Dieses lange Zeit sehr vitale Feld ist im letzten Jahrzehnt jedoch erlahmt, weil es auf empirische Forschung weitgehend verzichtet hat und das zentrale Dilemma nicht durch weitere theoretische Arbeiten aufgelöst werden konnte. Wir argumentieren, dass eine erneute Beschäftigung mit Agency jenseits der Agent-Struktur-Debatte für die Internationalen Beziehungen lohnenswert wäre, zumal von praxistheoretischen und materialistischen Theorien neue Impulse ausgehen. Hinzu kommt eine lebendige Auseinandersetzung mit Agency in verschiedenen empirischen Forschungsgebieten, wenn auch teils mit uneinheitlicher Terminologie - z. B. zur Agency von Internationalen Organisationen, von afrikanischen Staaten, von non-governmental organizations oder von Jugendlichen. Der Beitrag fasst den interdisziplinären Forschungsstand zusammen, entwickelt daraus Möglichkeiten für eine gemeinsame Agency-Forschung über ontologische Grenzen hinweg und zeichnet Konturen eines Forschungsfelds Agency in den Internationalen Beziehungen.
\end{abstract}

\section{Einleitung*}

1999 bezeichnete Colin Wight das Agent-Struktur-Problem als »totes Pferd«: »No matter how much one flogs them, some dead horses simply refuse to stay down « (Wight 1999: 109). Dass er wenige Jahre später ein umfangreiches Werk vorlegte, das dieses Problem theoretisch durchleuchtete (Wight 2006), verstehen wir als Anzeichen, dass dieses Pferd noch nicht so tot war, wie es Wight zunächst schien. Vielmehr ist es heute sogar wieder sehr lebendig, allerdings ohne sich dessen richtig bewusst zu sein.

Seit Wights apodiktischem Abgesang hat die Agency-Forschung eine Art Renaissance erlebt, allerdings weniger auf dem schon weitgehend abgegrasten Feld theoretischer Agent-Struktur-Debatten - auf dem Wights Buch von 2006 tatsächlich eine Art Endpunkt markiert -, sondern vielmehr auf anderen theoretischen Feldern, wie z. B. der Prinzipal-Agent-Theorie (Hawkins et al. 2006), der Praxistheorie (Bü-

* Eine frühere Version dieses Papiers wurde bei der Vierten Offenen Sektionstagung Internationale Politik der Deutschen Vereinigung für Politikwissenschaft (DVPW) in Magdeburg, 25. bis 27. September 2014, vorgestellt. Wir bedanken uns bei Jörg Langerwisch und Virginia Sroka für ihre Unterstützung bei der Arbeit an diesem Papier sowie bei Frank Gadinger, Aletta Mondré, Holger Niemann, der zib-Redaktion und drei anonymen Gutachter_innen für ihre hilfreichen Kommentare. 
ger/Gadinger 2008) sowie aktuell in Materialitätstheorien (Best/Walters 2013). Hinzu kommen empirische Arbeiten aus einer Reihe von Gebieten, z. B. zur Agency von Internationalen Organisationen (Barnett/Finnemore 2004), von afrikanischen Staaten (Brown/Harman 2013b), von non-governmental organizations (NGOs) (Tucker 2014) oder von Jugendlichen (Bøås 2013). Zwar verwenden diese Forschungsgebiete nicht alle den Agency-Begriff, meinen aber denselben Sachverhalt.

Wir sind der Ansicht, dass die Agency-Forschung in den Internationalen Beziehungen (IB) trotz dieser aktuellen Blüte ihr Potenzial bislang zu wenig entfaltet. Um dafür einen Impuls zu geben, stellen wir drei Thesen auf: Erstens existieren die verschiedenen ontologischen und empirischen Werke relativ unverbunden nebeneinander. Die empirischen Arbeiten nehmen kaum Bezug aufeinander und ihre Erkenntnisse fließen nicht in die ontologischen Diskussionen ein. Terminologie und Konzepte sind oft uneinheitlich. Im Gesamtresultat führt dies dazu, dass es keine Agency-Forschung als eigenständiges Forschungsfeld gibt. Wir halten dies für eine verpasste Chance.

Zweitens fassen wir Agency als ein Brückenkonzept auf, das sich dazu eignet, die fehlende Konversation zwischen ontologischen und empirischen Beiträgen anzustoßen. Um den Agency-Begriff für die Forschung fruchtbar zu machen, möchten wir derartige Konversationen ermöglichen und ein Forschungsfeld umreißen. Dadurch entstehen mehrere Vorteile: a) eine Agency-Perspektive kann einen neuen Blickwinkel auf bekannte empirische Phänomene bieten, indem unterschiedliche theoretische Perspektiven zusammengebracht werden wie z. B. beim Thema globale Diplomatie, zu dem u. a. die African-Agency-Literatur, Prinzipal-Agent-Theorie und Praxistheorie etwas zu sagen haben; b) der Rückgriff auf eine explizit gemachte Agency-Perspektive und einen möglichen gemeinsamen Agency-Begriff eröffnet Optionen für vergleichende Forschung in unterschiedlichen Kontexten, z. B. zur Frage wie Agenten ihre eigene Agency beeinflussen können; und c) dies ermöglicht eine Weiterentwicklung der ontologischen Literatur durch eine stärkere empirische Fundierung, was insbesondere für ein besseres Verständnis der Agent-Struktur-Beziehung hilfreich wäre.

Drittens wird der Agency-Begriff in der vorhandenen IB-Literatur sehr unterschiedlich verwendet. Daher lohnt sich ein Seitenblick in andere sozialwissenschaftliche Disziplinen, die sich zum Teil viel umfassender mit dem Thema Agency beschäftigen. Unserer Auffassung nach lassen sich hier einige Anstöße für eine Klärung der Begriffe finden, die zur Weiterentwicklung der Agency-Forschung in den IB beitragen können.

Basierend auf diesen Thesen plädieren wir in diesem Aufsatz für einen »ökumenischen« Umgang mit der Pluralität der Ansätze auf dem Feld der Agency-Forschung. Die verschiedenen Ansätze teilen gemeinsame Interessen, verfolgen diese jedoch auf der Grundlage von Prämissen, die sich nicht auf einen einheitlichen Rahmen reduzieren lassen. Gemeinsame Arbeit im Forschungsfeld Agency ist nur auf der Basis eines Dialogs zwischen den verschiedenen Ontologien möglich. Dies erfordert nicht zuletzt Transparenz über die eigenen Annahmen, begriffliche Stringenz und ein Bewusstsein dafür, wo die Schnittstellen zwischen verschiedenen An- 
sätzen liegen. Dazu soll dieser Artikel beitragen, indem er die vielfältigen Begriffsverständisse herausstellt und klärt sowie die Unterschiede zwischen den verschiedenen Ansätzen herausarbeitet.

Dazu stellen wir zunächst gängige theoretische Sichtweisen auf Agency dar, die in den IB anerkannt sind, und arbeiten die Unterschiede zwischen den verschiedenen Theorien heraus. Im dritten Abschnitt heben wir die Gemeinsamkeiten dieser unterschiedlichen Strömungen hervor, entwickeln Ansätze für Dialog zwischen den Paradigmen und situieren unseren eigenen Agency-Begriff in dieser Landschaft. Im vierten Abschnitt kartieren wir verschiedene Forschungsgebiete, die sich mit empirischen Phänomenen von Agency befassen z. B. zur Agency von Internationalen Organisationen, von afrikanischen Staaten, von NGOs oder von Jugendlichen. Dabei identifizieren wir Möglichkeiten zur Verknüpfung dieser Arbeiten und diskutieren methodologische Anforderungen an Agency-Forschung.

\section{Theoretische Sichtweisen auf Agency}

Es gibt eine Vielzahl theoretischer Beiträge zur Ontologie von Agency, die wir in diesem Abschnitt zu kartieren versuchen. Zunächst gehen wir dabei auf die AgentStruktur-Debatte ein, diskutieren danach praxistheoretische Zugänge zu Agency und wenden uns abschließend Beiträgen aus der IB-Rezeption der Actor-NetworkTheory (ANT) sowie von Assemblage-Theorien zu.

\subsection{Die Agent-Struktur-Debatte}

Die IB-Debatte um Agency, Akteure und ihre strukturelle Einbettung wurde stark durch soziologische Beiträge des Critical Realism (Bhaskar 1997), des Social Realism (Archer 1995; 2000) und der Strukturationstheorie (Giddens 1986) inspiriert. Gemeinsam ist diesen Ansätzen die Annahme, dass Akteure über Agency verfügen, diese aber keine inhärente Qualität eines Akteurs sei. Vielmehr fragen sie danach, wie Akteure ihre Handlungsmöglichkeiten im Zusammenspiel mit einschränkenden und ermöglichenden strukturellen Bedingungen ausüben können. Zudem teilen diese Ansätze eine nicht-reduktionistische und nicht-deterministische Sichtweise auf die Autonomie sozialer Strukturen. Agency und Struktur konstituierten sich stets gegenseitig und seien zudem von gleichem ontologischem Status.

Unter Agency versteht Giddens (1986: 9) sowohl die auf ein Individuum bezogene Handlungsfähigkeit, das heißt die Kapazitäten, Fähigkeiten und Potenziale zum Erkennen möglicher Handlungen, als auch die Möglichkeit, Handlungen tatsächlich intentional auszuüben (Handlungsmächtigkeit). Unabhängig von der tatsächlichen Ausführung einer Handlung geht er davon aus, dass das Individuum potenziell zur Handlung in der Lage gewesen wäre und charakterisiert Agency damit als eine exklusiv-menschliche Disposition und Eigenschaft. Strukturen seien in das Handeln selbst eingebettet und somit nichts dem Subjekt Äußeres, sondern etwas Inneres. 
Sie seien sowohl ermöglichend als auch beschränkend und damit rekursiv organisierte transformative Regeln und Ressourcen, nach denen Akteure in der Reproduktion ihres sozialen Lebens handeln und auf die sie sich dabei beziehen (Giddens 1986: 25). Hay (2002: 121) kritisiert, dass Giddens das Agent-Struktur-Dilemma nicht auf theoretischer, sondern nur auf definitorischer Ebene löse, indem er Handlungsfähigkeit, Handlungsmächtigkeit und Strukturen als etwas dem Subjekt Innewohnendes definiere, und plädiert dafür, das dialektische Zusammenspiel von Agency und Struktur in realen Kontexten sozialer und politischer Interaktion in den Mittelpunkt zu rücken (Hay 2002: 127).

Eine differenziertere Auseinandersetzung mit Agency bieten Emirbayer und Mische (1998), die sich für ein mehrdimensionales, relationales sowie inter- und transdisziplinär anschlussfähiges Agency-Verständnis aussprechen:

»We define it [menschliche Agency] as the temporally constructed engagement by actors of different structural environments - the temporal-relational contexts of action - which, through the interplay of habit, imagination, and judgment, both reduces and transforms those structures in interactive response to the problems posed by changing historical situations« (Emirbayer/Mische 1998: 970).

Sie verbinden vielfältige Handlungshintergründe und sozial eingebettete, relationale Handlungsdimensionen - Habitualisierung, das heißt Routinen, Traditionen, Erwartungen etc. (habit/iteration), absichtsvolles und planendes Handeln (judgement), Vorstellungen und normative bzw. planvolle/prospektive Ausrichtungen (imagination). Ferner differenzieren sie zwischen endogener und exogener Agency und verweisen auf die Temporalisierung, das heißt Raum- und Zeit-Relationalität sowie Konditionalität von Agency. Akteure leben simultan in verschiedensten zeitlichen Bezügen, gewichten diese aber situativ unterschiedlich, wobei es auch zu Widersprüchen zwischen den Perspektiven kommen kann.

Die IB haben sich mit dem Thema Agency zuerst in Form des Agent-StrukturProblems befasst. Dies hat kontroverse Debatten entfacht und zu einer notwendigen Beschäftigung mit Grundfragen internationaler Beziehungen geführt. Dennoch blieb die Diskussion über Agency in den IB bislang recht einseitig und sollte deshalb mit Blick auf eine Differenzierung und Systematisierung weitergeführt werden (Wight 2006: 178). Den Auftakt bildete ein Aufsatz von Alexander Wendt (1987), der sich mit der Frage beschäftigte, ob Staaten das internationale System konstituieren oder umgekehrt. Bereits vor Wendt hatten sich andere Autor_innen dieser Frage gewidmet (Wendt 1987: 338, Fn. 8), sein Beitrag blieb jedoch auf lange Zeit am einflussreichsten. Er geht davon aus, dass alle IB-Theorien zumindest implizit Aussagen zur ontologischen Priorität von Agenten oder Strukturen treffen (Wendt 1987: 337-340). Theorien, die den Schwerpunkt entweder auf Agenten oder Strukturen legen, unterstellt er eine fatale Unfähigkeit, die Eigenschaften ihrer primären Einheiten zu erklären. Mit Rekurs auf Giddens (1986) und Bhaskar (1997) argumentiert er, dass Agenten und Strukturen ontologisch gleichrangig seien und sich wechselseitig konstituierten. Zwar kritisiert Herborth (2004: 61-62), dass Wendt diesen Ansatz konzeptionell nicht durchhält, da er z. B. die kreativen Momente im Handeln der Akteure vernachlässige. Letztlich implizieren Wendts Ausführungen 
für die IB aber, dass sowohl strukturalistische als auch Akteurstheorien notwendig sind, um ein vollständiges Bild sozialer Prozesse zu zeichnen.

Wendts Artikel rief eine lebhafte und lang andauernde Auseinandersetzung mit dem Thema hervor, ohne das Agent-Struktur-Dilemma tatsächlich konzeptionell zu überwinden (s. die Übersichten bei Bieler/Morton 2001 und Wight 2006: 62-120). Viele Beiträge waren primär daran interessiert, das Agent-Struktur-Problem entweder zu negieren oder in eine der beiden Richtungen aufzulösen. Auch die Versuche, das Problem durch neogramscianische (Bieler/Morton 2001) oder poststrukturalistische Perspektiven (Doty 1997) fruchtbar zu machen, konnten sich nicht durchsetzen. Bieler und Morton (2001) bezeichneten das Agent-Struktur-Problem daher als gordischen Knoten für die IB-Theorie (vgl. ähnlich Wight 1999: 109).

Der letzte größere Beitrag in dieser Tradition kam von Wight mit seiner Monografie $»$ Agents, Structures and International Relations« (Wight 2006). Ähnlich wie Franke und Roos (2010), Herborth (2004) und Banerjee (2015), die sich aber anderer Quellen bedienen, spannt er einen weiten Bogen und führt die IB-Diskussion an ihre soziologischen und philosophischen Grundlagen zurück. Wight übt zwar deutliche Kritik an Wendt, bestätigt aber dessen zentralen Punkt, nämlich die unauflösliche Verbundenheit von Agent und Struktur:

$»$ At the same time as social structures are reproduced or transformed by human agency, they are also the very medium of this reproduction. This relationship, then, must be grasped as dynamic, not static, and hence not reductionist in either direction « (Wight 2006: 210).

Wights Verständnis von Agency beginnt beim Individuum, dessen Freiheit der Subjektivität moralisches Handeln erst möglich macht. Gleichzeitig verweist er darauf, dass ein Akteur im sozialen Leben auch ein Agent von etwas sein kann (Wight 2006: 206-211). Sozialen Kollektiven und Organisationen schreibt er eine besondere Form der Agency zu, da sie über keine eigene Intentionalität verfügen, die über die reine Aggregation der Intentionen ihrer Mitglieder hinausgeht (Wight 2006: 199-205). Praktisch agieren Organisationen darüber, dass sie Rollen bereitstellen, deren Inhaber_innen mit einer Agency der Organisation ausgestattet werden und in ihrem Namen sprechen und handeln. Franke und Roos werfen Wight in diesem Punkt Inkonsequenz vor: Obschon Wight den Fokus auf die Agency von Individuen lege, schreibe er Staaten und Organisationen dennoch eine aktive kausale Macht zu, auf Strukturen einzuwirken. Nach Franke und Roos können Staaten jedoch nicht als Personen betrachtet werden, sondern stellen Strukturen gemeinschaftlicher Praktiken dar (Franke/Roos 2010: 1064-1065).

Dieser und andere Beiträge zeigen weitere Aspekte auf, die die Grenzen der aktuellen Debatte verdeutlichen. Hervorzuheben ist insbesondere die Kritik, dass Wights Ansatz kaum Auskunft darüber gibt, wie sich Strukturationsprozesse ausgestalten (Franke/Roos 2010; ähnlich auch Banerjee 2015 und Herborth 2004). Franke und Roos (2010: 1067-1068) entwerfen dafür ein pragmatistisches Modell, wie Strukturen das Handeln von Agenten beeinflussen, z. B. indem sie Handlungsregeln erzeugen, Beziehungsmuster und Positionen von Agenten festlegen oder deren Handlungs(un)möglichkeiten definieren. Die Prozesse, die von Agenten durch ihre 
Handlungen innerhalb dieser Strukturen gemeinschaftlicher Praktiken ausgelöst werden, können durch die Transformation von Krisen und Problemen strukturellen Wandel auslösen.

Ähnlich stellt auch Herborth (2004) soziales Handeln von Agenten als Triebfeder offener Transformationsprozesse in den Mittelpunkt. Er unterscheidet dabei Handeln in Routine- und Problemsituationen - in ersteren verfolgen Agenten auf Erfahrungen basierende Handlungsweisen und reproduzieren dadurch Strukturen, während sie in Krisen- und Problemsituationen nicht auf Routinen und erprobte Handlungen zurückgreifen können. Bewähren sich neu entwickelte Verhaltensweisen in der Problemlösung, fließen sie in den Erfahrungsschatz des Subjekts ein (Herborth 2004: 79). Dieser Erfahrungsschatz ist, wie Banerjee (2015) argumentiert, nicht nur ein Handlungsrepertoire. Erfahrungen werden in autobiografische Narrative der Agenten integriert und manifestieren sich in ihren Identitäten, die wiederum bestimmte Handlungen und Praktiken hervorbringen. Durch diese emergenten Effekte sozialen Handelns werden wiederum Strukturen konstituiert.

In dieser Auseinandersetzung kam es trotz des angenommenen gleichen Status von Agenten und Strukturen häufig zu einer Rekonzeptionalisierung von Strukturen, während Agency als soziales Konstrukt aber eher vernachlässigt wurde (Hellmann et al. 2005: 145). So lässt sich zum einen z. B. weder bei Banerjee (2015) noch bei Herborth (2004) oder Franke und Roos (2010) eine explizite und ausführliche Auseinandersetzung mit dem Agency-Begriff finden. Zum anderen ist die unspezifische, z. T. synonyme Verwendung zentraler Begriffe (z. B. Hay 2002: 253; Franke/Roos 2010: 1068) wie z. B. die analytische Unterscheidung zwischen Agent und Akteur weiterhin eine Herausforderung. Bislang sind solche reflektierten Differenzierungen noch recht selten in der IB-Literatur zur Agent-Struktur-Debatte zu finden. Zu den Ausnahmen gehört Dessler (1989), der Agent und Akteur wie folgt differenziert: $\gg$ An actor can act socially only because there exists a social structure to draw upon, and it is only through the actions of agents that structure is reproduced (and potentially transformed)« (Dessler 1989: 452). Dieser Ansatz stellt einen deutlichen Bezug zu den strukturellen Hintergründen und der wechselseitigen Konstitution von Agent und Struktur her. Erst die Strukturen ermöglichen Akteuren soziales Handeln und machen in diesem Zuge Akteure zu Agenten.

Dennoch merkt Wight (2006: 199) kritisch an, dass damit nicht ausreichend klar wird, wer oder was Agent oder Struktur ist. In seiner Diskussion darüber, was es bedeutet, Agent zu sein, verweist Wight zum einen auf die »capacity to do«, zum anderen auf den Status einer Entität »as an agent of something« (Wight 2006: 212). Daraus entwickelt er letztlich drei Dimensionen von Agency: 1) die individuelle Agency des menschlichen Subjekts bzw. dessen aus persönlichen Eigenschaften entstehende Handlungsfähigkeit, 2) die Agency, die den Agenten aus dem sozialen System und den Kollektiven und Gruppen, zu denen sie gehören, erwächst, sowie 3) die Agency, die durch das Einnehmen sozialer Positionen bzw. das Ausfüllen konkreter Rollen entsteht (Wight 2006: 212-215). Brown (2012: 1895) übersetzt diese Systematik wie folgt: In der ersten Dimension geht es um individuelle Kapazitäten - wie viel subjektive Handlungsfähigkeit hat ein Akteur und was tut er da- 
mit? In der zweiten Dimension wird der Akteur ein Agent einer sozialen Totalität, gewissermaßen ein »Träger« seiner Umwelt. In der dritten Dimension wird der Akteur in formellen oder informellen sozialen Rollen positioniert (z. B. Diplomat_in, Anführer_in, Demonstrant_in), aus denen weitere Agency erwachsen oder verwehrt werden kann.

\subsection{Praxistheoritische Perspektiven auf Agency}

Praxistheorien rücken praktisches Wissens und Alltagshandeln ins Zentrum ihres Interesses. Für Praxistheorien bilden soziale Praktiken - mit anderen geteiltes Tun, Sprechen, Fühlen und Denken -, deren Verankerung in Körpern und Artefakten sowie ihre Verknüpfung zu Wissensbeständen die kleinste Einheit des Sozialen (Reckwitz 2008: 188, 191). Durch diese Ontologie bieten sie einen anderen Zugang zu Agency als handlungsbezogene Agent-Struktur-Theorien. Praxistheorie ist in den IB inzwischen eine durchaus etablierte Strömung, die nicht für eine einheitliche Theorie des Sozialen steht, sondern für eine Familie an Ansätzen, die bestimmte ontologische Annahmen teilen (Büger/Gadinger 2014; Adler/Pouliot 2011). Die Beschäftigung der IB mit praxistheoretischen Fragen reiht sich in den practice turn anderer geistes- und sozialwissenschaftlichen Disziplinen ein (Schatzki et al. 2001); insofern könnte Praxistheorie das Potenzial haben, einen gemeinsamen methodischen Zugang zur Erforschung von Agency zu bieten und damit zum Bindeglied einer multidisziplinären Agency-Forschung zu werden.

Praktiken waren bereits in Giddens' Strukturationstheorie zu finden und erhielten Eingang in die IB durch dessen Rezeption z. B. von Wendt (1999). Im ursprünglichen Ansatz wirken Praktiken als vermittelndes Element in der Konstitution zwischen Agent und Struktur (Doty 1997). Allerdings kam Praktiken zunächst kein eigener ontologischer Stellenwert zu, vielmehr wurden sie als kausaler Mechanismus verstanden, über den Strukturen auf Agenten wirken und Agenten Strukturen reproduzieren (Büger/Gadinger 2014: 5). Die Praxistheorie in den IB beruft sich dagegen stärker auf die sozialtheoretischen Arbeiten von Bourdieu (1977), Boltanski (2012), Reckwitz (2002), die Actor-Network-Theory von Latour (2005) sowie die wegweisenden Studien von Neumann (2002) sowie Adler und Pouliot (2011).

Durch den Fokus auf soziale Praktiken liegt der Schwerpunkt von Praxistheorien nicht auf der Ebene der Handlungskoordination zwischen Akteuren, sondern darauf, was Akteure dazu bringt, die Welt als geordnet anzusehen und deshalb handlungsfähig zu werden. Es geht also nicht mehr darum, warum einzelne Akteure handeln, sondern um die routinisierten Handlungsmuster eines Kollektivs, die im sozialen Raum zu beobachten sind. Diese Handlungsmuster erhalten ihren Sinn durch implizites Wissen und sind materiell in Körpern und Artefakten verankert (Bongaerts 2007: 249).

Praxistheoretiker_innen wie Büger und Gadinger $(2014: 3$, 99) sehen das Potenzial dieser Herangehensweise darin, traditionelle Dualismen wie zwischen Struktur und Agency zu vermeiden und zu überwinden. Ähnlich drücken dies Adler und 
Pouliot aus: $\gg(\mathrm{P})$ ractice forces us to engage with the relationship between agency and the social and natural environments, with both material and discursive factors, and with the simultaneous processes of stability and change" (Adler/Pouliot 2011: 2). Aus praxistheoretischer Sicht wäre es ein Fehlschluss, das Individuum mit Agency und das Soziale mit Struktur gleichzusetzen. Praktiken bestehen bereits vor der Handlung des Subjekts, sie ermöglichen Handeln, strukturieren und begrenzen es aber auch. Praktiken werden von Agenten inszeniert und tragen gleichzeitig zum framing der Agenten bei, die dadurch wissen, wer sie sind und welche sozialen Erwartungen es an ihre Rollen gibt. Sie sind also unabhängig und zugleich abhängig vom Subjekt. Kurz gesagt: »Practices create agents and give meaning to agency « (Adler/Pouliot 2011: 23). Praktiken verwandeln strukturelles Hintergrundwissen in sozial bedeutungsvolle Handlungen. Struktur wird damit ein sozial anerkannter Verhaltensstandard, der Praktiken wiedererkennbar macht. Insofern muss der Dualismus von Agent und Struktur dahingehend erweitert werden, dass sowohl Agency als auch Struktur gemeinsam Praktiken konstituieren und ermöglichen (Adler/ Pouliot 2011: 16).

\subsection{Actor-Network-Theory und Assemblage-Theorien}

Eine andere Herangehensweise wählen Zugänge wie die Actor-Network Theory (ANT) oder kulturwissenschaftliche Theorien der Materialität (Latour 2005), ebenso wie praxistheoretisch ausgerichtete sozialtheoretische Beiträge zur Materialität (z. B. Schatzki 2010) sowie feministische Anstöße zum »new materialism« (z. B. Alaimo/Hekman 2008), in dem Materie ihren passiven Charakter verliert und aktiv an der Konfiguration der Welt beteiligt ist.

Die neue Rolle von Materialität schließt in den IB an Kritiken an, wonach materielle Faktoren in den vorhandenen Ansätzen gegenüber ideellen Faktoren stiefmütterlich behandelt werden (Voelkner 2015). In der ANT stehen sich Ideen und Materie nicht als separate Kategorien gegenüber, sondern werden in Hybriden kombiniert. Das traditionelle Verständnis des Sozialen als Beziehung zwischen Menschen wird durch Verbindungen aus menschlichen und nichtmenschlichen Wesen ersetzt. Die ANT betont das Handlungspotenzial von Gegenständen, sieht diese aber nicht getrennt von Menschen, sondern als Teil handlungsermöglichender Netzwerke. In diesem Netzwerk werden Identitäten geformt und Praktiken deutlich oder ermöglicht. Die »Hybridwelt« (Latour 2000: 27) ist ein Netzwerk aus Technik, Dingen, Menschen, Riten und Symbolen, die einander beeinflussen und die soziale Welt formen (Latour 2010: 19, 112).

In dieser Denkweise wird Agency als eine Handlungskraft verstanden, die als Effekt der Beziehungen zwischen menschlichen und nichtmenschlichen Wesen entsteht. Danach haben auch Dinge das Potenzial, Effekte auf das tägliche Handeln auszulösen. Sie sind aber von bloßen Objekten zu unterscheiden, indem Dinge das Verhalten von Menschen lenken und so an der Konstruktion der sozialen Welt mitwirken. Im Gegensatz zu Objekten verweist der Begriff des Dings auf einen Kon- 
fliktstoff, da Dinge »[...] ermächtigen, ermöglichen, anbieten, ermutigen, erlauben, nahelegen, beeinflussen, verhindern, autorisieren, ausschließen und so fort « (Latour 2010: 124) können.

Wenn sich durch die An- oder Abwesenheit von Dingen etwas ändert, besitzen sie Wirkungskraft (Agency) und werden von Latour als »Aktanten« (Handlungsträger) bezeichnet. Dieser aus der Semiotik entlehnte Begriff soll das Ding als Handlungsträger vom Menschen unterscheiden, um es damit von der alltagssprachlichen Verwendung des Begriffes Akteur zu differenzieren (Latour 2000: 219). Einen nennenswerten Unterschied zwischen menschlichen Akteuren und nicht-menschlichen Aktanten möchte Latour damit aber nicht unterstellen, schon gar kein ontologisches Primat des Akteurshandelns: »Akteur ist wer von vielen anderen zum Handeln gebracht wird « (Latour 2010: 81). Hiermit positioniert sich Latour deutlich gegen klassische Handlungstheorien, die den Menschen als autonomen Schöpfer seiner Entscheidungen begreifen, und weist den intentionalistischen Handlungsbegriff der traditionellen Soziologie zurück, wonach nur Menschen Handlungen vollziehen können. Handeln bedeutet für ihn, dass etwas »durch eine Folge von elementaren Transformationen [...] andere Akteure modifiziert« (Latour 2001: 108).

Die IB-Rezeption der ANT ist zurzeit noch im Entstehen. Während einige Beiträge das Potenzial der ANT hervorheben (Best/Walters 2013; Büger 2013), weisen andere auf bislang noch unbeantwortete Fragen hin (Nexon/Pouliot 2013). Zu Letzteren gehört auch Barry (2013), der noch viel Aufwand darin erkennt, die ANT für die IB zu übersetzen. Er kritisiert insbesondere, dass bisherige ANT-Arbeiten dem historischen Kontext und den strukturellen Hintergründen eines Akteur-Netzwerks relativ wenig Aufmerksamkeit schenken. Umgekehrt argumentiert Büger (2013), dass die ANT die Chance biete, sich aus strikten Dualismen wie Agency/Struktur zu befreien, und hebt die Familienähnlichkeiten zwischen ANT, Praxistheorie und pragmatistischen Ansätzen in den IB hervor.

Theorien von Assemblage verstehen wir als eine besondere Spielart materialistischer Theorien, die einen ähnlichen Ansatz wählen wie die ANT (zu Unterschieden und Ähnlichkeiten vgl. Acuto/Curtis 2014a: 5). Assemblage meint »[ein] kontingentes Ensemble von Praktiken und Gegenständen, zwischen denen unterschieden werden kann [und] die entlang den Achsen von Territorialität und Entterritorialisierung ausgerichtet [sind] (Deleuze/Guattari 1987: 504-505).

Der Assemblage-Begriff wurde insbesondere von Acuto und Curtis (2014b) für die IB fruchtbar gemacht. Die zentrale Annahme von Assemblage ist, dass nicht nur Menschen Agency haben, sondern auch Dinge, Tiere, Maschinen etc., die sich in kontigenter Weise zusammensetzen (Büger 2014: 61). Assemblage-Theorien befassen sich mit der Frage, wo Agency zu finden ist. Dies wird bereits im von Deleuze und Guattari im Original verwendeten Begriff des agencement deutlich, welcher darauf hindeutet, dass der Zusammenschluss von Dingen in einer Assemblage dieser Agency verleiht (Müller 2015: 28). Agency wird also vom Akteur getrennt und als auf die gesamte Assemblage verteilt angesehen (Acuto/Curtis 2014a: 8). Diese verteilte Agency wird z. B. im Bereich der Klimaforschung deutlich, wo Individuen und Gruppen, aber auch technologische Werkzeuge, Messinstrumente und Compu- 
termodelle eine Assemblage bilden, die eine gemeinsame Agency besitzt. Gegenüber dem Strukturbegriff nehmen Assemblage-Theorien eine skeptischere Haltung ein als Praxistheorien, da sie anstatt der Stabilität von Wissensbeständen und Bedeutungen eher von »emergence, heterogeneity, the decentred and the ephemeral in nonetheless ordered social life« (Marcus/Saka 2006: 101) sprechen.

\subsection{Die Unmöglichkeit eines übergreifenden Agency-Ansatzes}

Im Vergleich dieser Zugänge werden erhebliche Unterschiede ihrer jeweiligen Agency-Begriffe deutlich. Diese Differenzen liegen insbesondere in den AgencyDefinitionen, die sich aus unterschiedlichen Ontologien speisen, im Stellenwert von Intentionalität sowie in den verwendeten Machtbegriffen.

Für Agent-Struktur-Theorien und Praxistheorien bedeutet Agency, dass menschliche Akteure die Fähigkeit und Kapazität besitzen, Handlungsmöglichkeiten zu erkennen und diese bewusst und intentional auszuüben. Die ANT und AssemblageTheorien verstehen Agency als Potenzial, Effekte auf Handeln auszuüben. Während Agency also traditionell als Handlungsfähigkeit verstanden wird, bedeutet es für materialistische Ansätze eher Wirkungskraft und die Möglichkeit, in Interaktion zu treten.

In ihrer Ontologie beruhen Agent-Struktur-Theorien auf klassischen Handlungstheorien, die den Menschen (oder eine Gruppe) als handelndes Subjekt sehen. Dinge sind hier nur insoweit relevant, als sie vom menschlichen Akteur zur Durchführung seiner Handlungen verwendet werden, haben aber keinen eigenen ontologischen Status. Die ANT und Assemblage-Theorien geben Dingen, Symbolen und Riten einen eigenen ontologischen Status und behandeln diese über den Aktantenbegriff als gleichrangig mit menschlichen Akteuren. Praxistheoretiker_innen gehen hier einen Mittelweg - sie betonen die Körperlichkeit von Praktiken, die in einen materiellen Kontext eingebettet sind und darüber einen Bezug zu Dingen haben. Ähnlich wie die ANT erkennt auch die Praxistheorie an, dass Dinge Effekte auf Handlungen haben, und sieht sich daher als Brücke zwischen idealistischen und materialistischen Ontologien (Büger/Gadinger 2014: 74). Dennoch räumen die meisten Praxistheorien Dingen nicht dieselbe Akteursqualität ein wie es die ANT tut. Die Praxistheorie scheint bei aller Anerkennung der Bedeutung von Materialität noch stärker dem menschlichen handelnden Subjekt verhaftet als Latour. Nicht zuletzt wird in der ANT und in Assemblage-Theorien Agency vom individuellen Aktanten gelöst: Die Assemblage oder das Akteur-Netzwerk verfügt über Agency (Mattissek/Wiertz 2014: 162).

Darüber hinaus unterscheiden sich die Ansätze auch erheblich in ihren Strukturbegriffen. Während die Struktur bei Giddens und in Praxistheorien einen relativ stabilen Charakter hat (allerdings bei ersterem einen eher institutionalistischen, bei letzterem einen stärker wissensbezogenen Einschlag), wird in Assemblage-Theorien eher der transitorische Charakter von Strukturen betont. Praxistheorie hat daher auch einige Ideen zum Verständnis der Agent-Struktur-Beziehung beizusteuern, 
welche bei Giddens und anderen Agent-Struktur-Ansätzen jenseits der Prämisse der Ko-Konstitution relativ unerforscht bleiben. Praxistheorie fügt durch das Element der Praxis noch einen dritten Bestandteil hinzu, der zwischen den beiden Polen moderiert, aber auch einen eigenen ontologischen Status hat.

Diese Ontologien unterscheiden sich deutlich im Stellenwert, den sie Bewusstsein und Intentionalität zuschreiben. In klassischen Handlungstheorien ist Intentionalität eine Voraussetzung für Handeln und damit für die Ausübung von Agency. Bongaerts (2007) problematisiert jedoch die implizite Annahme bewusster Intentionalität und argumentiert, dass Intention nur einen Teilausschnitt sozial relevanter Tätigkeitsmodi abbildet. Gewohnheiten seien damit ausgeschlossen, obwohl diese im sozialen Kontext äußerst bedeutungsvoll seien. Ähnlich argumentieren Praxistheorien, die den Fokus von Handlungsmotiven hin zu Handlungsmustern verschieben. In materialistischen Theorien spielt Intentionalität gar keine Rolle mehr, da dort zum einen auch nicht-bewussten Dingen eine Handlungsfähigkeit zugeschrieben wird, zum anderen die Wirkung von Agency eher emergent ist und somit nicht auf Zielvorstellungen einzelner Akteure zurückgeführt werden kann (Müller 2015: 30).

Nicht zuletzt ergeben sich aus den genannten Unterschieden auch völlig unterschiedliche Machtkonzepte. In Agent-Struktur-Theorien ist Agency nicht das gleiche wie Macht, obwohl beides akteursbezogene Konzepte sind (Wight 2006: 206). Macht wird dabei als Kapazität zur Durchsetzung von Interessen verstanden, sei es durch Befehl und überlegene Gewalt, durch Einfluss in Institutionen oder die Manipulationen der Präferenzen anderer Akteure. Demgegenüber ist Agency die viel fundamentalere Kapazität, in einem Kontext überhaupt Sinn zu konstruieren, Interessen zu formulieren und nach ihrer Erfüllung streben zu können. Freilich kann ein Akteur ohne Agency auch keine Macht ausüben, ein Akteur ohne Macht kann aber dennoch über Agency verfügen.

In der Praxistheorie wird Macht demgegenüber »nicht als eine übertragbare Substanz oder Ressource begriffen, sondern praxisimmanent verstanden. Dies bedeutet, Macht mit heterogenen Relationen zu identifizieren, die beständiger Aufrechterhaltung in der Praxis bedürfen« (Schäfer 2016: 150). In der ANT gelten Aktanten als mächtig, wenn sie viele Beziehungen aufrechterhalten, die Beziehungen im AkteurNetzwerk strukturieren sowie Rollen und Skripte für andere Aktanten definieren (Büger/Gadinger 2014: 47-48). Kritiker_innen werfen der ANT oft vor, dass die Theorie dafür blind sei, wie strukturelle Machtunterschiede die Möglichkeiten von Aktanten einschränken, Beziehungen zu knüpfen und sich im Akteur-Netzwerk zu positionieren (Müller 2015: 30). Assemblage-Theorien knüpfen eher an von Foucault inspirierte poststrukturalistische Machtkonzepte an, betonen aber deren materielle Dimension (Mattissek/Wiertz 2014: 162).

Dieser Abschnitt machte deutlich, dass es nicht möglich ist, einen übergreifenden Agency-Ansatz zu entwickeln, der die verschiedenen Herangehensweisen unter einem Dach vereint. Grundsätzliche Streitfragen sind 1) wer oder was ein Agent ist und 2) was es bedeutet, Agency auszuüben. Für die erste Frage wäre eine Systematik möglich, die Akteure als Spezialfall eines großen Universums von Aktanten versteht. Allerdings bezweifeln wir, dass dies für Assemblage-Theorien und die ANT 
tragbar ist, die Wert auf die kollektive Agency der Assemblage bzw. des Netzwerks anstatt ihrer konstitutiven Elemente legen. Ferner müssten klassische Handlungstheorien dafür akzeptieren, dass Intentionalität keine unbedingte Voraussetzung für Agency darstellt, sondern nur bei einer bestimmten Klasse von Agenten zu finden ist. Und letztlich müsste die ANT eine Unterscheidung von dinglichen Aktanten und menschlichen Akteuren akzeptieren, die Latour zwar auf alltagssprachlicher Ebene anerkennt, die aber in der ANT explizit abgelehnt wird. In der zweiten Frage ist der Dissens noch fundamentaler. Während manche Theorien Agency als Handlungsfähigkeit verstehen, bedeutet sie für andere Wirkungskraft und wiederum für andere die Fähigkeit, Interaktionen einzugehen. Eine gemeinsame Systematik scheint in dieser Frage unmöglich.

\section{Möglichkeiten des Dialogs in der Agency-Forschung}

\subsection{Gemeinsamkeiten und Chance eines ökumenischen Dialogs}

Trotz dieser Unterschiede behalten wir unsere Position bei, dass Agency ein mächtiges Brückenkonzept sein kann, das viele verschiedene Forschungsfragen verbindet. Insofern verfolgen wir weiterhin den Anspruch, ein »Forschungsfeld Agency« zu umreißen.

Wir plädieren für eine »ökumenische« Herangehensweise an Agency-Forschung, um uns dem geteilten Ziel anzunähern, das Phänomen Agency besser zu verstehen. Neben den bereits genannten Unterschieden teilen die verschiedenen Ansätze eine Reihe von Gemeinsamkeiten, die einen Dialog ermöglichen und ergiebig machen. Erstens lehnen alle Ansätze strukturalistische Erklärungen des Sozialen ab. In jeder Ontologie sind Agenten - was auch immer darunter verstanden wird - zumindest teilweise dafür verantwortlich, wie soziale Prozesse ablaufen und ausgehen. Zweitens folgt daraus eine Würdigung der Kontingenz dieser Prozesse. Drittens teilen Agency-Theorien ein Interesse an den Mikro- und Mesodynamiken des Sozialen und sind skeptisch gegenüber Versuchen, aus diesen Dynamiken auf Makrologiken eines Systems zu schließen. Ein Dialog auf Grundlage dieser Gemeinsamkeiten könnte unter den Bedingungen einer transparenten Darstellung der eigenen ontologischen und theoretischen Annahmen und einem daraus hervorgehenden genau definierten und stringent eingesetzten Vokabular gelingen. Damit ließen sich Schnittstellen der verschiedenen Ansätze leichter ergründen und Arbeiten könnten gegebenenfalls explizit aufeinander Bezug nehmen.

\subsection{Unser Agency-Ansatz}

Im Sinne der von uns formulierten Forderung nach einer transparenten Darstellung der eigenen ontologischen und theoretischen Annahmen stellen wir in diesem Ab- 
schnitt unseren eigenen Ansatz zur Agency-Forschung vor. Unser Ansatz bewegt sich in der Tradition der Agent-Struktur-Debatte und wir gehen davon aus, dass diese auch von Anregungen aus der Praxistheorie und aus materialistischen Theorien profitieren kann. Diese Ausrichtung erscheint uns über das größte Potenzial dahingehend zu verfügen, für mehrere der empirischen Forschungsgebiete zu Agency (s. nächster Abschnitt) eine gemeinsame theoretische Basis zu bieten. Wir erheben damit aber dezidiert nicht den Anspruch, den gemeinsamen konzeptuellen Ansatz für die IB zu skizzieren oder auf einen verengenden Agency-Begriff zu insistieren. Vielmehr wollen wir unseren Agency-Ansatz exemplarisch darlegen, um uns in der Pluralität zu verorten und damit für einen kritischen aber produktiven Dialog zu öffnen. Wir nehmen dabei begründet Bezug auf einige der vorgestellten Sichtweisen und grenzen uns an anderen Stellen von ihnen ab.

Unserer Definition nach ist Agency die sich strategisch-relational aus einem komplexen Beziehungsgefüge ergebende, ressourcenabhängige sowie raum- und zeitbeeinflusste, bewusste Handlungsfähigkeit und Handlungsmächtigkeit eines Agenten. Dieses Agency-Verständnis ist auf ein breites Spektrum von Akteuren wie z. B. Individuen, Gruppen, Institutionen, Korporationen, Kollektive oder Netzwerke anwendbar. Im Folgenden gehen wir einzeln auf die Elemente dieser Definition ein.

In unserem Ansatz werden Akteure zu Agenten durch ihre Fähigkeit zum bewussten (Nicht-)Handeln in Auseinandersetzung mit Strukturen und anderen Akteuren. Wir sehen ebenso wie Hay vor allem im Bewusstsein bzw. im bewussten (Nicht-)Handeln das entscheidende Kriterium zur Differenzierung von Akteur und Agent: »Agency refers to action, in our case to political conduct. It can be defined, simply, as the ability or capacity of an actor to act consciously and, in so doing, to attempt his or her intentions « (Hay 2002: 94). Instinktives, habituelles oder automatisiertes Handeln wäre nach dieser Auffassung kein Ausdruck von Agency, zumal dieses Handeln lediglich Strukturen reproduziert.

Agency umfasst sowohl die individuelle Handlungsfähigkeit, das heißt die Kapazität zum Erkennen und Bewerten möglicher Handlungen, als auch Handlungsmächtigkeit, das heißt die Fähigkeit zum intentionalen Umsetzen von Handlungsstrategien. Agency verstehen wir daher als die Kapazität eines Akteurs, Entscheidungen autonom zu treffen und nach ihrer Umsetzung zu streben. Akteure werden dabei aber nicht als atomistische Individuen verstanden, deren Motive und Überlegungen rein endogen zu verstehen sind. Vielmehr entwickeln Akteure diese in Auseinandersetzung mit ihrer Umwelt in konkreten sozialen Interaktionen und Kontexten. Dies betont die zeitlich dimensionierte Verwobenheit von Akteuren in unterschiedlichen strukturellen Kontexten (Emirbayer/Mische 1998). Zur Differenzierung bietet sich das Agency-Konzept von Wight und Brown an, das drei Quellen von Agency unterscheidet. Demnach erlangen Akteure Agency (1) durch verschiedene Formen individueller Handlungsfähigkeit, sowie (2) durch den sozialen Hintergrund und (3) durch aus sozialen Rollen konstruierte Handlungsmächtigkeiten. Agency ist dabei - wie oben diskutiert - nicht mit Macht gleichzusetzen, welche impliziert, dass ein Agent mit seinen Handlungen das intendierte Ziel auch gegen Widerstände erreichen kann. 
Unser Verständnis von Agency setzt voraus, dass Akteure intentional handeln können. Dies ist insbesondere bei kollektivem Handeln zu beachten, wo nicht jedes Beispiel gleich eine kollektive Agency nach sich zieht, z. B. wenn ein spontaner Zusammenschluss von Twitter-Nutzer_innen "\#bringbackourgirls« fordert. Unser Verständnis von Intentionalität ist breit und bedeutet lediglich, dass Akteure Präferenzen formulieren und Strategien entwickeln können, um diese Präferenzen mit ihrem Handeln zu verfolgen. In diesem Verständnis verfügen auch korporative Akteure über Intentionalität, solange sie in der Lage sind, Entscheidungen zu treffen und nach ihrer Umsetzung zu streben. Dies tun beispielsweise Organisationen durch interne Strukturen und Prozesse, die das individuelle Handeln von Akteuren miteinander verknüpfen.

Agency ist zeitlich und räumlich kontextuell, das heißt sie entsteht im Kontext von Strukturen und Beziehungsgeflechten zwischen Akteuren. Wir orientieren uns an einem breiten Strukturbegriff nach Giddens und verstehen darunter nicht abstrakte Muster sozialer Beziehungen und Phänomene, sondern etwas in dem Handeln selbst Eingebettetes und dem Subjekt Inneres (kritisch: Wight 2006: 121-176). Strukturen erfassen sowohl rekursiv organisierte Regeln, die nicht nur formalisiert und mechanisch charakterisiert sind, sondern auch Routinen und Verhaltensweisen (Giddens 1986: 21; Herborth 2004), sowie Ressourcen, nach denen Akteure handeln und auf welche sie sich beziehen. Sie sind sowohl beschränkend als auch ermöglichend und schwerlich selbst beobachtbar.

Praktiken stellen das Scharnier zwischen Struktur und Agency dar. Hier folgen wir dem Praxisbegriff von Adler und Pouliot, ohne deshalb deren Fokus auf Praktiken zu übernehmen:

»Practices translate structural background intersubjective knowledge into intentional acts and endow them with social meaning. Structure, in turn, shows up in practices in the form of standards of competence that are socially recognized. [...]. >Suspended $<$ between structures and agency, practices are simultaneously enacted (agency) and inserted within a social context or political order (structure)« (Adler/Pouliot 2011: 16).

Wir sehen Agency in konkrete und über die Zeit gewachsene Strukturen eingebettet. Insofern setzen wir die gegenseitige Konstitution von Agency und Struktur als gegeben voraus. Innerhalb dessen ist es jedoch möglich, in seiner Forschungsperspektive Akzente zu setzen, um den Blickwinkel zu variieren. So legt Brown (2012) einen Fokus auf die Agency afrikanischer Staaten, um damit den Pessimismus der afrikabezogenen IB-Forschung herauszufordern und gegen den Strich zu bürsten (vgl. auch Zanotti 2013).

In einem relationalen Verständnis konstituieren, transformieren und prägen sich Agency und Struktur gegenseitig. Unserer Auffassung nach handelt es sich dabei um ein dialektisches Verhältnis, das heißt die Kategorien werden synthetisiert und existieren simultan in jeder Situation mit- und nebeneinander, ohne einer Seite eine ontologische Priorität einzuräumen. Durch den Fokus auf das dialektische Zusammenspiel in realen Kontexten sozialer und politischer Interaktion kann dabei der Dualismus zwischen Agency und Struktur überwunden werden (Hay 2002: 127). Diese Perspektive ist für die IB naheliegend, da in ihnen zumeist kollektive und 
korporative Akteure betrachtet werden, deren Handlungsfähigkeit und Handlungsmächtigkeit, ja sogar deren »Akteurhaftigkeit« erst durch die sozialen Spielregeln der internationalen Beziehungen geschaffen werden. Während auf diese Weise Strukturen Agency konstituieren, verändern Agenten die Strukturen.

Am Beispiel des Staates lässt sich die Ko-Konstitution von Struktur und Agent verdeutlichen. Brown (2012: 1898-1899) beschreibt, wie die Agency von Staaten durch den Akt der Anerkennung konstruiert wird. Die gegenseitige Anerkennung von Souveränität definiert die Mitgliedschaft im internationalen »Club« der Staaten (Griffiths 2017); mit ihr gehen eine Reihe von Praktiken wie z. B. diplomatische Kontakte oder Staatsbesuche einher. Diejenigen Agenten, die als Repräsentanten eines souveränen Staates in den sozialen Kontexten internationaler Politik auftreten, erhalten damit ein Recht, für diese Staaten zu sprechen und zu handeln. Darüber hinaus sind sie ermächtigt, andere Staaten und deren Repräsentant_innen anzuerkennen. Für nichtstaatliche Akteure sind diese Regeln weniger klar, deshalb auch stärker im Fluss, aber aus diesen Gründen letztlich umso interessanter. Die Agency des/der Piraten/in, des/der Terroristen/in, des/des Traders/in und des/der Sprechers/in einer zivilgesellschaftlichen Initiative aus dem globalen Süden hat sich in den letzten Jahrzehnten zum Teil drastisch gewandelt (Avant et al. 2010). Gleichzeitig gibt es einen klaren Mangel an empirischer Forschung darüber, wie diese Agency entsteht und was die Akteure selbst tun (können), um deren Ausmaß und Gehalt zu beeinflussen (O’Neill et al. 2004: 170; Podder 2015: 42-43).

Agency wird jedoch nicht nur durch Strukturen geprägt, sondern auch im Kontext von Beziehungsgefügen zwischen Akteuren. Anders als in materialistischen Theorien verbleibt in unserem Ansatz Agency weiterhin bei den menschlichen Agenten und wird nicht auf die Assemblage oder das Akteur-Netzwerk transferiert. Gleichwohl übernehmen wir den relationalen Charakter von Agency als eine wichtige Ergänzung der klassischen Agent-Struktur-Beziehung.

Alle Akteure im internationalen System benötigen Ressourcen, um ihre Agency auch realisieren zu können, das heißt um ihre abstrakte Handlungsfähigkeit auch in konkrete Handlungsmächtigkeit umzusetzen. Agency im internationalen System ist sehr voraussetzungsvoll und basiert auf Anerkennungsprozessen, die sich auf Regeln, Skripte, Normen und Diskurse beziehen und den Agenten bestimmte, teils routinisierte Verhaltensweisen aufzwingen. Dies verdeutlicht das Zusammenwirken von Beziehungsgeflechten und Strukturen sowie deren Ausdruck in Praktiken der Produktion von Agency. Jenseits dieser immateriellen Ressourcen benötigen Agenten z. B. auch interne Organisationskapazitäten, Zugriff auf bestimmte Formen von Humankapital sowie finanzielle Mittel, um sich in politische Prozesse einbringen zu können. Dabei kann die Dinglichkeit dieser Ressourcen, jenseits ihrer bloßen Verteilung, wichtige handlungsermöglichende und -beschränkende Wirkungen auf die Parameter von Handeln und Nichthandeln haben. So hatte die Größe des Konferenzzimmers des Generalsekretärs der World Trade Organization (WTO) den Effekt, dass am sogenannten Green-Room-Verfahren, einem wichtigen informellen Abstimmungsprozess, nur ca. 20 bis 40 Delegationen beteiligt werden konnten. Unser Fokus auf Handlungen und Bewusstsein macht es aber unmöglich, Dingen (oder 
Aktanten in der Sprache der ANT) eine eigene Agency, losgelöst von der ihrer Schöpfer_innen und Nutzer_innen, zuzuschreiben.

Die Ressourcendimension von Agency wird maßgeblich durch die globale Ungleichheit bedingt (Lambach 2017), was sich sowohl auf materielle als auch immaterielle Ressourcen bezieht (Brown/Harman 2013a: 8-10). So können schwache institutionelle Kapazität und innenpolitische Instabilität vieler afrikanischer Staaten deren Handlungsmächtigkeit einschränken. Dies hat auch Folgen für die Fähigkeit von Akteuren, ihre Agency auszuüben oder notfalls auch selbst zu erstreiten. Hilfreich ist hier die Unterscheidung zwischen taktischer und strategischer Agency, die Honwana (2006) aus der Handlungstheorie von de Certeau (1988) entwickelt hat. Darin ist strategische Agency die Fähigkeit, seine Umwelt zu gestalten und sich von dieser unabhängig zu machen. Demgegenüber ist taktische Agency das situationsabhängige Kalkül der Machtlosen, die sich in Räumen zurechtfinden müssen, die andere geschaffen haben.

Gerade diese Ungleichheit macht eine Erforschung von Agency so relevant. Dadurch kann gefragt werden, wie die Machtlosen in diesem System ihre taktische Agency nutzen und wie sie die Spielregeln der vorgegebenen Struktur zum eigenen Vorteil wenden können. Beispielsweise beschreibt Fisher (2013), dass das System globaler Entwicklungszusammenarbeit (EZ) davon abhängig ist, dass die Empfängerländer die offiziellen EZ-Narrative der Geberländer zumindest rhetorisch akzeptieren. Zwar können die Empfängerländer den Gehalt dieser Narrative kaum beeinflussen, aber Agency über die Auswahl und Akzentuierung des Narrativs ausüben. Dieses und weitere Beispiele (Williams 2013: 136-141; Brown/Harman 2013a: 10) legen nahe, dass die Agency-Forschung Ungleichheit im internationalen System verstärkt berücksichtigen sollte. Dies gilt insbesondere für die Auseinandersetzung mit empirischen Fragestellungen, die wir im nächsten Abschnitt diskutieren.

\section{Konturen eines Forschungsfelds »Agency»}

Wir haben argumentiert, dass eine kollaborative Agency-Forschung, auch über ontologische Gräben hinweg, neue Möglichkeiten eröffnet. Zum einen können wir dadurch das Phänomen Agency besser fassen, zum anderen kann eine Agency-Perspektive neue Impulse für etablierte Forschungsgebiete bringen. Weiterhin plädieren wir für eine verstärkt empirische Auseinandersetzung mit Agency. Es gibt bereits umfassende, aber voneinander isolierte Forschungen $\mathrm{zu}$ Agency in verschiedenen Kontexten, die wir hier näher vorstellen möchten. Wir konzentrieren uns dabei auf die am weitesten entwickelten Forschungsgebiete mit einem klaren Agency-Bezug, nämlich:

- African Agency,

- die Agency Internationaler Organisationen sowie deren moralische Agency,

- die Agency von NGOs,

- die Agency globalisierungskritischer Bewegungen, 
- die Agency von Jugendlichen in transnationalen Kontexten, z. B. im Rahmen des »Arabischen Frühlings« und in Nachkriegssituationen,

- Forschung zu diplomatischen Praktiken, sowie

- kritische Sicherheitsforschung, die die Rolle von Technologie als Aktant in komplexen Sicherheitsnetzwerken herausarbeitet.

Damit möchten wir nicht unterstellen, dass in anderen Forschungsgebieten keine Beschäftigung mit Agency stattfindet, sondern lediglich, dass dies weniger stark geschieht und keine feldinternen Debatten zu Agency nach sieht zieht. Zum Beispiel haben O’Neill, Balsiger und van Deveer Vorschläge für die Kooperationsforschung vorgestellt, wie empirische Forschung zum Agent-Struktur-Problem aussehen könnte, z. B. zur zunehmenden Agency nichtstaatlicher Akteure sowie zur Hypothese, dass sich Agency durch Kooperation zwischen Akteuren verändern kann (O’Neill et al. 2004: 169-170). Die Mehrzahl dieser Gebiete - mit Ausnahme der praxistheoretischen Sicherheitsforschung und materialistischer Ansätze - arbeitet mit Agent-Struktur-Theorien und ist somit anschlussfähig an unser unter 3.2. skizziertes Konzept von Agency.

\subsection{Bestehende Forschungsgebiete}

Die genannten Forschungsgebiete werden in diesem Abschnitt dargestellt, um Konturen eines möglichen Forschungsfelds zu zeichnen, dessen Entstehen wir mit diesem Beitrag voranbringen möchten. Anschließend stellen wir einige Überlegungen zu möglichen Verknüpfungen zwischen den Gebieten und den dabei entstehenden methodischen Herausforderungen an, um damit das brückenbauende Potenzial eines Agency-Ansatzes anzudeuten.

Die Beschäftigung mit der Agency der Staaten des subsaharischen Afrikas (African Agency) bewegt sich in einem auf Wight (2006) aufbauenden Verständnis von Agency. Brown (2012; Brown/Harman 2013a) nutzt diesen Zugang, um die Position afrikanischer Staaten in der globalen Diplomatie neu zu interpretieren. Er sieht den Wert einer Agency-Perspektive darin, dass sie die klassische Sichtweise herausfordert, wonach afrikanische Staaten nur passive Opfer externer Kontrolle seien. Angesichts der zunehmenden Handlungsmächtigkeit afrikanischer Akteure möchte Brown stattdessen ein differenzierteres Bild Afrikas zeichnen, das zwischen AfroPessismus und übertriebenem Optimismus liegt (Brown/Harman 2013b: 1; ähnlich Beswick/Hammerstad 2013: 477). Er erkennt an, dass sich afrikanische Agency innerhalb von »tight corners« (Brown/Harman 2013a: 1), also innerhalb deutlicher struktureller Einschränkungen bewegt, z. B. dem Einfluss von Großmächten, nachteiligen Wirtschaftsstrukturen oder globalen Diskursen, die Afrika die Fähigkeit zur Selbstbestimmung absprechen. Dennoch fragt er, wie afrikanische Akteure das internationale System beeinflussen können, wie sie darin handeln, wann sie sich anpassen und wann dagegen auflehnen (skeptisch: Williams 2013). In diesem Sinne befasst sich eine Reihe von Beiträgen mit der Nuanciertheit der Taktiken, mit denen 
afrikanische Akteure ihrer Marginalisierung begegnen. ${ }^{1}$ Dazu greifen z. B. Beswick und Hammerstad (2013) auf Bayarts (2000) Konzept der Extraversion zurück. Damit beschreiben sie einerseits den Beitrag afrikanischer Akteure zu ihrer eigenen Marginalisierung, andererseits heben sie aber auch die Spielräume hervor, die sich in dieser Position der »Unterordnung« ergeben. Andere untersuchen die Beziehungen Afrikas zu den westlichen Industriestaaten als Prinzipal-Agent-Beziehung (Fisher 2013).

Die Forschung zur Agency Internationaler Organisationen (IOs) arbeitete lange Zeit in erster Linie mit der Prinzipal-Agent-Theorie. ${ }^{2}$ Dabei geht es um die Fragen, warum Staaten Kompetenzen an IOs delegieren und wie Staaten den Umgang von IOs mit diesen Kompetenzen kontrollieren (Haftel/Thompson 2006; Hawkins et al. 2006). Der Begriff Agency wird dabei zumeist zugunsten von Konzepten wie Autonomie vermieden, auch wenn deren Bedeutung letztlich sehr ähnlich ist. Die Agen$c y$ einer IO wird durch das Delegationsverhältnis konstituiert, was aber nicht bedeutet, dass IOs deshalb nur passive Befehlsempfänger_innen ihrer staatlichen Prinzipale sind. Stattdessen unternehmen Organisationen häufig Versuche, ihre Autonomie auszuweiten, z. B. indem sie Staaten davon überzeugen weitere Aufgaben zu delegieren (Hawkins/Jacoby 2006: 199-202) oder interne Reformprozesse kreativ gestalten. So stellen Barnett und Finnemore fest:

»IOs are active agents in their own change. States did not command the IMF [International Monetary Fund] to adopt a more ambitious policy regarding balance-of-payments difficulties; IMF staff were involved in making sense of past failures and argued for new policy directions as a way of trying to fulfill their basic mandates « (Barnett/Finnemore 2004: 158).

Auf diese Weise können sich Organisationen verselbständigen und eine Agency entwickeln, die von ihren Schöpfern nicht vorgesehen war. Koch (2009) bietet für diesen Prozess der »autonomization « eine systemtheoretische Erklärung. ${ }^{3}$ Für Koch ist es das Ziel einer IO, ihre Umwelt so zu gestalten, dass sie vorhersagbarer wird und sich Ungewissheiten für die Organisation und ihre Mitglieder reduzieren (Koch 2009: 438).

Eng mit der IO-Forschung sind auch Arbeiten zur Moral Agency Internationaler Organisationen verknüpft. Das grundsätzliche Konzept moralischer Agency wird insbesondere in der Theologie, Philosophie, Psychologie und den Neurowissenschaften behandelt (Christen 2010; Cushman 2008; Paharia et al. 2009), aber auch für die IB fruchtbar gemacht, indem nach der moralischen Verantwortung bzw. Entscheidungsfähigkeit internationaler Institutionen gefragt wird (Erskine 2003b). In den IB ist die Bezugnahme auf Moral Agency zunehmend bei Fragen der Verant-

1 Vgl. die Beiträge in Brown/Harman (2013b) und in einer Sonderausgabe der Zeitschrift »Conflict, Security \& Development « (Beswick/Hammerstad 2013 und Weitere).

2 Die Prinzipal-Agent-Theorie wurde in der Wirtschaftswissenschaft entwickelt, um stabile, asymmetrische Beziehungen zu beschreiben, bei der ein Prinzipal einen Agenten mit dem Erbringen einer Handlung beauftragt und damit Autorität delegiert (Jensen/Meckling 1976: 308).

3 Vgl. z. B. Haftel/Thompson (2006) und Schindler (2014) für vergleichbare Versuche aus anderen theoretischen Blickwinkeln. 
wortung sowie nach Rechten und Pflichten beobachtbar. Auf der globalen Ebene gibt es inner- und außerhalb der Vereinten Nationen eine Vielzahl an Handlungsaufforderungen, Verantwortungszuschreibungen und Schuldzuweisungen - zum Beispiel in Bezug auf die Responsibility to Protect (Roff 2011), Menschenrechte (Alston 2000), Global Governance (Held/Koenig-Archibugi 2007) und Weltarmut (Mieth 2012). Die Literatur dazu benennt Moral Agency häufig nicht als solche, sondern behandelt sie implizit und zieht sie für die jeweilige Argumentation heran. Erskines (2003a) Modell der »institutional moral agency« zur Erklärung der (ethischen) Grundlagen von Verantwortung bzw. deren Zuschreibung mit besonderem Bezug auf Institutionen scheint vielversprechend zur Behandlung derartiger Fragen. Zahlreiche Autor_innen haben sich mit Bezug auf Erskines Modell mit der Frage befasst, wann internationale Akteure auf Krisen reagieren sollten, wie erfolgreich sie dabei waren und warum sie notwendige Reaktionen bei Katastrophen vermissen ließen (Barnett 2003).

Auch zur Agency nichtstaatlicher Akteure gibt es eine Reihe unterschiedlicher Beiträge, auch wenn Lewis und Opoku-Mensah (2006) beklagen, dass der Agency von NGOs zu wenig Aufmerksamkeit zuteil werde. Seckinelgin (2006) bietet dafür eine erste Annäherung aus einer von Giddens inspirierten Perspektive: »The analysis of NGO capabilities needs to be located within social processes implicit in multiple institutionalised settings that differently frame NGO identity and agency « (Seckinelgin 2006: 720). Diese Einbettung von NGOs in institutionalisierte Kontexte wird von Tucker (2014) mit dem Begriff der Subjektivierung beschrieben. Sie stellt dar, wie Diskursregime und Wahrheitskonfigurationen die Vorstellungen von IO-Mitarbeiter_innen und Politikentscheider_innen darüber formen, wie NGO-Vertreter_innen angemessen auftreten sollen. Danach müssen NGOs ihre taktische Agency im Idealfall so gestalten, dass sie in einem umschriebenen Rahmen durch Angemessenheit ihres Auftretens, Verhaltens und Sprechens Wirkung erzielen können (Tucker 2014: 382-384).

Die Literatur zur globalisierungskritischen Bewegung (alter-globalization movement) hebt die besondere Agency dieses »movement of movements « (Gibson-Graham 2006: xix) hervor. Diese Meta-Bewegung besteht aus einer Vielzahl lokaler und transnationaler Initiativen und wurde von Hardt und Negri (2004) mit dem populären Schlagwort der multitude beschrieben. Im Unterschied zu stärker integrierten Bewegungen ist das alter-globalization movement relativ unkoordiniert, nicht institutionalisiert und verfügt über keine identifizierbaren Sprecher_innen. Seine Willensbildung erfolgt dezentral sowie auf Treffen wie dem Weltsozialforum. Gill sah hier optimistisch »new potentials and forms of global political agency (Gill 2000: 137), jedoch kritisieren Stephen (2009) und Gibson (2008), dass es die Bewegung nie geschafft habe, klassische Probleme kollektiven Handelns zu überwinden. Demgegenüber argumentieren Scholl und Freyberg-Inan (2013), dass das Scheitern der Bewegung weniger an internen Ursachen, sondern vielmehr an der Stärke der hegemonialen Orthodoxie lag, die sich gegen Kritik abschottete, diese kooptierte oder versicherheitlichte. Mit ihrem neogramscianischen Ansatz bieten 
Scholl und Freyberg-Inan ein weiteres Beispiel dafür, inwieweit die Etablierung von Diskursen ein wichtiges Mittel für Akteure ist, um eigene Agency zu stärken.

Nicht zuletzt zeigt auch die Forschung zur Agency Jugendlicher (Youth Agency), wie marginalisierte Gruppen handlungsfähig werden können. Die Literatur zu Youth Agency befasst sich mit Jugendlichen, die durch ihre Beteiligung an grenzüberschreitenden Bewegungen und Netzwerken in vielen Ländern des globalen Südens als politisch relevante und mobilisierbare Gruppe angesehen werden. Gleichzeitig befinden sich Jugendliche angesichts mangelnder wirtschaftlicher Perspektiven und starrer gesellschaftlicher Strukturen oft in einer benachteiligten und marginalisierten Stellung. Dies wird jedoch von einer zunehmenden Zahl von Jugendbewegungen und -organisationen herausgefordert, die mehr Mitsprache verlangen und über transnationale Diffusionsprozesse voneinander lernen (Honwana/De Boeck 2005). Durch ihre Beteiligung an revolutionären Prozessen wie dem »Arabischen Frühling« erhalten Jugendliche auch in den IB folgerichtig mehr Aufmerksamkeit (z. B. Gertel et al. 2014). Es geht aber nicht nur um die politische Mobilisierung von Jugendlichen in Form von Bewegungen - Youth Agency zeigt sich auch darin, wie Jugendliche damit umgehen, Gegenstand der Politik zu sein, wie es z. B. Bøås (2013) anhand der alltäglichen Überlebensstrategien von Jugendlichen in (Nach-)Kriegskontexten untersucht. Ganz im Sinne von Honwanas (2006) Unterscheidung von taktischer und strategischer Agency zeigt er, wie sich Youth Agency im Streben nach einem »Ausbrechen« aus desolaten Lebensumständen ausdrückt, wie sich Jugendliche improvisierend und adaptierend an wechselnde Bedingungen, Schicksalsschläge und existenzielle Herausforderungen anpassen, die in hohem Maß von zufälligen externen Faktoren bestimmt werden und damit von den Jugendlichen wenig vorhersehbar und nicht kontrollierbar sind (ähnlich Podder 2015).

In der Diplomatieforschung gibt es einen sehr aktiven Bereich praxistheoretischer Arbeiten (Pouliot/Cornut 2015). Diese Arbeiten sprechen oft nur indirekt über Agency, da ihr Fokus auf den Praktiken liegt, die Agency und Struktur miteinander verbinden. Daher bieten diese Arbeiten hilfreiche Erkenntnisse über die Konstitution von Agenten und wie diese durch Praktiken mitgestaltet wird. So demonstrieren Adler-Nissen und Pouliot (2014), wie Praktiken der Machtausübung im diplomatischen Spiel der Vereinten Nationen die Rolle von Diplomat_innen mit Bedeutung füllen. Adlers (2008) Arbeit zur Formation und Expansion sicherheitspolitischer Praxisgemeinschaften zeigt, wie Praktiken in Institutionen verankert werden und darüber wiederum das Verhalten von Staaten beeinflussen.

Ähnliche Beiträge finden sich auch in der kritischen Sicherheitsforschung. Die praxistheoretische Forschung zum Thema der Grenzsicherung untersucht Praktiken der Grenzkontrolle zwischen Sicherheitsdiskursen, offiziellen Politiken und dem Alltagshandeln der Grenzschützer_innen (Côté-Boucher et al. 2014; Bigo 2002). Materialistische Ansätze bieten beispielsweise Arbeiten zu security assemblages, die sich häufig mit der Rolle privater Akteure für die Herstellung von Sicherheit beschäftigen (Abrahamsen/Williams 2009). Empfehlenswert ist besonders die Studie von Schouten (2014) zur Sicherheit am Flughafen Amsterdam-Schiphol, der konsequent dingliche Aktanten (Körperscanner, Flüssigkeiten) und Sicherheitsap- 
parate mit deren menschlichem Personal als Akteur-Netzwerk untersucht. Ferner bieten Studien zu Kampfdrohnen und Überwachungssystemen interessantes Anschauungsmaterial zu Möglichkeiten der ANT und Assemblage-Theorien. So schreibt Leander Drohnen eine »technological agency« (Leander 2013) als Aktanten in einem komplexen Netzwerk von Menschen, Organisationen, Technologien und Gesetzen zu, das zu einer Hybridisierung von unterschiedlichen Rechtsgebieten im Feld des Drohnenkriegs geführt habe. Auch für Holmqvist haben Drohnensysteme »agentic capacities« (Coole 2005) als Teil von »human-material assemblages« (Holmqvist 2013: 539). Diese Kapazitäten sind auch politisch, da sie z. B. über algorithmische Mustererkennung Ziele identifizieren und klassifizieren und damit Informationen über die Gefahrenlage für ihre menschlichen Pilot_innen vorstrukturieren (Holmqvist 2013: 544-545). In seiner Untersuchung israelischer Drohnenangriffe demonstriert Walters (2014) die politische Relevanz technischer Details, z. B. von typischen Sprengmustern eines Waffensystems, und grenzt damit seine materialistische Vorgehensweise von sprach- und diskursfokussierten Ansätzen ab. Rothe (2015) verbindet diese beiden Richtungen in seiner Analyse davon, welchen Einfluss Technologien wie das EU-Erdbeobachtungssystem »Copernicus« auf die Versicherheitlichung des Klimawandels haben. Er argumentiert, das Verständnis von verteilter Handlungsmacht in materialistischen Theorien biete die Chance, »das Problem von agency in diskurstheoretischen Ansätzen wie dem der Versicherheitlichung neu zu denken« (Rothe 2015: 108). Agency liegt danach nicht bei den Entscheidungsträger_innen, sondern ist »über eine breite Assemblage an Akteuren, Technologien und Diskursen verteilt« (Rothe 2015: 119). Ferner zeigt Côté (2016) in einer Metaanalyse, dass die Versicherheitlichungsforschung die Agency des Adressat_innenkreises eines versicherheitlichenden Sprechakts trotz dessen zentraler theoretischer Rolle unterschätzt, und demonstriert damit sehr anschaulich, wie eine klarere Beachtung des Themas Agency zur Weiterentwicklung etablierter Forschungsgebiete und Theorien beitragen kann.

\subsection{Berührungspunkte bisheriger Forschung und Perspektiven für die Zukunft}

Aus der Querbetrachtung der verschiedenen Forschungsgebiete wird deutlich, dass in vielen empirischen Gebieten eine lebhafte Beschäftigung mit Agency stattfindet. Dabei bleibt es bislang jedoch bei Debatten innerhalb dieser Gebiete; für eine genuine Agency-Forschung fehlt es an einer Verknüpfung über thematische Grenzen hinweg. Dies liegt nur zum Teil an unterschiedlichen Ontologien materialistischer und akteurstheoretischer Ansätze, da die meisten Gebiete für akteurstheoretische Agent-Struktur-Zugänge anschlussfähig sind. Das Hauptproblem ist vielmehr ein fehlendes Bewusstsein für die bereits existierende Vielfalt laufender Forschung.

Diese Gebiete ließen sich mit Agency als Brückenkonzept fruchtbar miteinander verbinden, was wiederum der Forschung innerhalb der einzelnen Forschungsgebiete wichtige Impulse verleihen könnte. Wir möchten dies am Beispiel der Arbeiten zu African Agency, zu NGO-Agency und zu Youth Agency verdeutlichen. Zwischen 
diesen Forschungsgebieten gibt es deutliche Ähnlichkeiten, jedoch verweist keiner der oben genannten Texte auf Literatur aus den beiden anderen Gebieten. Thematisch geht es in allen drei Forschungsgebieten um die Agency von Agenten, die nur wenig Kontrolle über die hierarchischen Verhältnisse haben, in denen sie leben. Diese ließe sich z. B. unter dem aus der Foucault'schen Sozialtheorie entlehnten, aber bislang noch nicht näher definierten Begriff der »agency of the governed « (Stern et al. 2015) zusammenbringen. Gemeint ist damit die Fähigkeit von Menschen zum eigenständigen Handeln, obwohl sie in vielfältigen Verhältnissen des Gesteuert-Werdens oder Selbst-Steuerns verstrickt sind: »People are clearly not only passive victims to the relations of rule to which they are subjected « (Stern et al. 2015: 1).

Dies ist unmittelbar anschlussfähig an den Begriff der taktischen Agency (aus der Youth-Agency-Forschung), der insbesondere die Kreativität in den alltäglichen, existenziellen Herausforderungen der Subjekte betont. Mit diesem Begriff ließen sich Verhaltensweisen afrikanischer Staaten beschreiben, die in internationalen Kontexten auch oft wenig Kontrolle über die Umstände ihres Handelns haben. Mit dem in der African-Agency-Literatur verwendeten Begriff der Extraversion ließe sich umgekehrt zeigen, wie die gesteuerten Subjekte durch ihre Nach-Außen-Orientiertheit an der Reproduktion ungleicher Strukturen mitwirken, obgleich dies aus ihrer Binnenperspektive natürlich eine vollkommen rationale, ja teils alternativlose Handlungsweise ist. Dies trifft einerseits auf Jugendliche zu, lässt sich aber auch auf NGOs übertragen, die oft stark vom Wohlwollen mächtiger Geber oder einflussreicher Akteure abhängig sind und an deren Wünschen sie sich oft orientieren (müssen). Hier bietet sich ein hilfreicher Kontrast zwischen dem Extraversionsbegriff und dem Konzept der Subjektivierung aus dem Bereich der NGO-Agency-Forschung an: Während ersterer sich auf die bewussten Handlungen des Subjekts konzentriert, beschreibt letzterer das komplexe und subtile Geflecht an Diskursen und Normen, die Agenten über Internalisierung aufnehmen und an denen sie die Gestaltung ihrer Subjektivität orientieren. Dieser Mechanismus tritt natürlich ebenso wirkmächtig in Arenen globaler Diplomatie auf, wo Repräsentant_innen afrikanischer Staaten sich ebenfalls im Geflecht persistenter Wissensbestände des comme il faut bewegen.

Jenseits dieses Beispiels gibt es weitere Möglichkeiten, innerhalb derer Agency sein Brückenpotenzial ausspielen kann. So könnte auch die Prinzipal-Agent-Theorie für weitere Problembereiche fruchtbar gemacht werden, z. B. für African Agen$c y$. Ebenso wie in der Forschung zu IO-Agency geht es dort um die Handlungsfähigkeit von untergeordneten Agenten, die nach eigener Autonomie streben. Diese Unterordnung besteht für afrikanische Staaten zwar nicht formell, aber sehr wohl informell (Lambach 2017).

Über mehrere Forschungsgebiete hinweg stellt sich die Frage, wie Agenten ihre eigene Agency beeinflussen können. Dabei müssen sowohl die internen Dynamiken von kollektiven bzw. korporativen Akteuren als auch deren externe Rahmenbedingungen in Betracht gezogen werden. Intern müssen Akteure Kohäsion herstellen, um überhaupt kollektive Intentionalität und Handlungsfähigkeit herauszubilden. 
Nach außen müssen sie die zur Verfügung stehenden Ressourcen nutzen, um Anerkennung einzufordern, ihre Spielräume zu nutzen und schrittweise zu vergrößern. Die Forschung zu IO-Agency ebenso wie die Literatur zum alter-globalization movement haben sich bereits mit dem Zusammenwirken dieser beiden Ebenen beschäftigt und können damit als Inspiration für andere Gebiete dienen. Aber auch sie können noch dazulernen, z. B. von der Forschung zu sozialen Bewegungen und zu Mobilisierung, um typische Muster kollektiven Handelns noch besser zu verstehen (zur Einführung: della Porta/Diani 2006).

Materialistische und praxistheoretische Ansätze befassen sich zwar teils mit anderen Fragestellungen als akteurstheoretische Arbeiten, aber auch hier ist gegenseitiges Lernen möglich. Globale Diplomatie wird sowohl praxistheoretisch als auch von den Forschungen zu African Agency, IO-Agency und NGO-Agency untersucht. Das Zusammenwirken von Akteuren, Technologien und Diskursen, z. B. in der Vernetzung von Jugendbewegungen und im alter-globalization movement bietet interessante Zugänge für ANT-Perspektiven. Auch bei der Frage nach den für Handlungsfähigkeit notwendigen Ressourcen, die in akteurstheoretischen Ansätzen bislang kaum systematisch erforscht wird, könnten materialistische Beiträge interessante Impulse liefern.

Letzten Endes ist die Agency-Forschung zu Agency als einem nicht nur theoretischen, sondern auch empirischen Konzept selbst noch eine Leerstelle. Wir haben bisher keine systematischen Erkenntnisse darüber, wie das Streben nach Agency mit Herrschaft und Widerstand interagiert, welche Taktiken Akteure dabei verfolgen oder wie dominante Akteure die Agency anderer Akteure manipulieren können. Es fehlt hier bislang eine Aggregation des gesammelten Wissens aus den unterschiedlichen Gebieten, um die stark ontologisch betriebene Agency-Diskussion voranzubringen. Auf diesem Wege, und mit weiteren Seitenblicken in andere Disziplinen, könnte man einer Antwort auf die Frage näherkommen, was Agency eigentlich ist.

Somit könnte ein Forschungsfeld entstehen, das andere Felder mit unterschiedlichen Analyseebenen, Gegenständen und Ontologien miteinander verknüpft. Hilfreich wäre dabei eine stärkere Systematik in der Terminologie - einzelne der oben genannten Forschungsgebiete vermeiden bislang den Agency-Begriff, meinen aber dasselbe. Es geht uns dabei nicht um die Etablierung eines vereinheitlichten Sprachrahmens, wir möchten aber auf das Potenzial hinweisen, das durch eine explizite Verortung in den vorhandenen Theoriegebäuden entsteht. Die Agency-Literatur ist pluralistisch und wird dies auch bleiben; dadurch bietet sie aber vielfältige Anknüpfungsmöglichkeiten für unterschiedliche Perspektiven.

\subsection{Methodologische Implikationen}

Die Frage, wie Agency erforscht werden soll, ist bislang nur wenig diskutiert worden (Wight 2006: 255). In akteurstheoretischen Ansätzen ist die zentrale Herausforderung, dass man die Ko-Konstitution von Agent und Struktur nicht gleichzeitig erforschen kann, sondern immer nur in Ausschnitten (Wendt 1987: 362-365; Dessler 
1989), was Wight (2006: 287) als »Ausklammern« (bracketing) bezeichnet. Für ein vollständiges Bild des Agent-Struktur-Verhältnisses muss man im Forschungsprozess zwischen agentenzentrierten und strukturalistischen Erklärungen hin- und herwechseln und das jeweils andere zumindest zeitweise als gegeben betrachten. Dies ist naturgemäß anspruchsvoll sowie zeit- und ressourcenintensiv.

Wo dies nicht möglich ist, besteht eine Alternative darin, einen Teilaspekt dieses komplexen Verhältnisses zu untersuchen und somit aus der Agent-Struktur-Beziehung zu abstrahieren. Allerdings muss dabei beachtet werden, dass z. B. eine aus einer agentenzentrierten Untersuchung gewonnene Erklärung auch wieder mit strukturalistischen Erklärungen zusammengebracht werden kann (Dessler 1989: 443-444, Fn. 412). Wight resümiert daher:

»What is crucial is that any complete explanation or large-scale research programme should explain, rather than assume, the nature of agency and structure, whereas any particular research endeavour must bracket out some of the attributes of these entities at some point in time « (Wight 2006: 288).

Ein Beispiel für dieses Vorgehen wäre die African-Agency-Literatur, die ihren Fokus auf Agenten legt, weil sie deren Verhalten im Rahmen gegebener struktureller Kontexte aus einer weniger afro-pessimistischen Perspektive verstehen möchte als die bisherige Literatur zu afrikanischen Staaten in den IB.

Für die meisten Zusammenhänge ist es sicher angemessen, qualitative Ansätze zu wählen, da sich Agency-Forschung i. d. R. mit einer Vielzahl von Variablen, Aspekten oder Zusammenhängen beschäftigt. Für bestimmte Fragestellungen sind aber auch quantative Herangehensweisen denkbar. So kann z. B. Weiler (2012) zeigen, dass die Größe einer Verhandlungsdelegation keinen signifikanten Einfluss darauf hat, wie viele seiner Ziele ein Staat im Rahmen von Klimaverhandlungen durchsetzen kann.

Für qualitative/interpretative Agency-Forschung bietet sich das ganze Spektrum möglicher Methoden an. Hier ist Bügers und Gadingers (2008: 294-296) Ansatz zu praxistheoretischer Methodik hilfreich, die eine kreative und undogmatische Methodenwahl nahelegt. Dabei heben sie den Wert ethnographischer Methoden hervor, da diese die größte Nähe zum Untersuchungsgegenstand erlauben, aber auch Formen von Beobachtung, Interviews und Textanalyse gehören zum üblichen Repertoire (Büger/Gadinger 2014: 84-86). Diese Überlegungen sind für die Agency-Forschung, die sich oft mit Mikro-Kontexten beschäftigt, sicherlich ebenfalls anwendbar. Letztlich ist aber eine systematische Verbindung des »Ausklammerns« spezifischer Attribute der Untersuchungsentitäten mit Fragen des Forschungsdesigns eine Herausforderung, der sich die Agency-Forschung noch genauer widmen muss.

\section{Fazit}

Die verschiedensten Disziplinen beschäftigen sich mit Fragen der Agency. Auch für die IB ist dies ein relevantes und fruchtbares Themengebiet, das aber bisher nur zum Teil erschlossen ist. Theoretische und empirische Zugänge gibt es viele, aller- 
dings existieren diese Literaturen bislang weitgehend unverbunden nebeneinander. Eine Verknüpfung dieser verschiedenen Forschung könnte große Potenziale erzeugen.

In diesem Aufsatz haben wir anhand von drei Thesen dargelegt, wie eine neue Agency-Forschung aussehen sollte. Erstens halten wir Agency für ein vielversprechendes Brückenkonzept, das zahllose Forschungsfragen und -gebiete produktiv miteinander verbinden kann. Dies unterstreichen auch Jackson und Nexon (2013) in ihrem Überblick über den Stand der IB-Theorie. Sie argumentieren, dass die Auseinandersetzung darüber, zu welchem Grad Akteure als autonom von ihrer Umgebung verstanden werden müssen, eine zentrale Diskussion der IB in der Phase nach den »großen Debatten« der Vergangenheit sein könnte.

Zweitens war die Behandlung von Agency in den IB lange zu sehr auf ontologische Fragen des Agent-Struktur-Problems fixiert. Durch empirische Arbeiten in unterschiedlichen Feldern sowie durch das Aufkommen praxistheoretischer und materialistischer Ansätze können die älteren Debatten mit neuem Leben gefüllt werden. Beispielsweise könnten damit neue Erkenntnisse über den bislang kaum verstandenen Prozess der Ko-Konstitution von Agent und Struktur entstehen. Eine empirisch reichhaltigere Agency-Forschung ist daher nicht nur für die Arbeit an einzelnen Forschungsthemen relevant, sondern kann auch zu fundierten Erkenntnissen zu einer der zentralsten Fragen der Agency-Forschung führen, nämlich was Agency selbst eigentlich ist.

Drittens zeigt ein Blick in andere Disziplinen einen deutlich fortgeschritteneren und nuancierteren Umgang mit dem Thema Agency. Einerseits können diese Beiträge zu einer Revitalisierung der IB-Diskussion herangezogen werden. Andererseits kann die IB durch die explizite Beschäftigung mit Agency ihren Teil zu einem emergenten transdisziplinären Forschungsfeld beitragen.

Dafür muss der Ausgangspunkt in einem pluralistischen und zugleich transparenten Umgang mit dem Agency-Begriff liegen. Handlungstheoretische, praxistheoretische und materialistische Ansätze haben unterschiedliche Ontologien und die methodischen Herausforderungen sind nicht zu unterschätzen, insbesondere für einen Dialog zwischen verschiedenen Paradigmen. Gleichwohl gibt es auch nennenswerte Gemeinsamkeiten: Erstens lehnen alle strukturalistische Erklärungen des Sozialen ab. Zweitens folgt daraus eine Würdigung der Kontingenz dieser Prozesse. Drittens teilen Agency-Theorien ein Interesse an den Mikro- und Mesodynamiken des Sozialen und sind skeptisch gegenüber Versuchen, aus diesen Dynamiken auf Makrologiken eines Systems zu schließen.

Bei gleichzeitiger Anerkennung der Unterschiede sehen wir das Potenzial für eine »ökumenische« Herangehensweise an Agency-Forschung. Die Verwendung von Agency als Brückenkonzept bietet aus unserer Sicht mehrere Vorteile: Erstens bringt sie die Forschung auf den einzelnen Forschungsgebieten voran, indem sie Impulse, Ideen und Konzepte aus anderen Gebieten einführt. Zweitens lassen sich vergleichbare Fragen an unterschiedliche Forschungsgegenstände stellen und aus dieser vergleichenden Perspektive Erkenntnisse über die Kontextbedingungen kau- 
saler Aussagen gewinnen. Drittens ließe sich dadurch mehr Systematik in der Terminologie durchsetzen, was die Dialogfähigkeit weiter vertieft.

\section{Literatur}

Abrahamsen, Rita/Williams, Michael C. 2009: Security Beyond the State. Global Security Assemblages in International Politics, in: International Political Sociology 3: 1, 1-17.

Acuto, Michele/Curtis, Simon 2014a: Assemblage Thinking and International Relations, in: Acuto, Michele/Curtis, Simon (Hrsg.): Reassembling International Theory. Assemblage Thinking and International Relations, New York, NY, 1-16.

Acuto, Michele/Curtis, Simon (Hrsg.) 2014b: Reassembling International Theory. Assemblage Thinking and International Relations, New York, NY.

Adler-Nissen, Rebecca/Pouliot, Vincent 2014: Power in Practice: Negotiating the International Intervention in Libya, in: European Journal of International Relations 20: 4, 889-911.

Adler, Emanuel 2008: The Spread of Security Communities: Communities of Practice, Selfrestraint, and NATO's Post-Cold War Transformation, in: European Journal of International Relations 14: 2, 195-230.

Adler, Emanuel/Pouliot, Vincent 2011: International Practices, in: International Theory 3: 1, $1-36$.

Alaimo, Stacy/Hekman, Susan J. 2008: Material Feminisms, Bloomington.

Alston, Philip 2000: Towards a Human Rights Accountability Index, in: Journal of Human Development 1: 2, 249-271.

Archer, Margaret Scotford 1995: Realist Social Theory. The Morphogenetic Approach, Cambridge.

Archer, Margaret Scotford 2000: Being Human. The Problem of Agency, Cambridge.

Avant, Deborah D./Finnemore, Martha/Sell, Susan K. (Hrsg.) 2010: Who Governs the Globe?, Cambridge.

Banerjee, Sanjoy 2015: Rules, Agency, and International Structuration, in: International Studies Review 17: 2, 274-297.

Barnett, Michael 2003: Eyewitness to a Genocide. The United Nations and Rwanda, Ithaca, NY.

Barnett, Michael/Finnemore, Martha 2004: Rules for the World: International Organizations in Global Politics, Ithaca, NY.

Barry, Andrew 2013: The Translation Zone: Between Actor-Network Theory and International Relations, in: Millennium 41: 3, 413-429.

Bayart, Jean-Francois 2000: Africa in the World: A History of Extraversion, in: African Affairs 99: 395, 217-267.

Best, Jacqueline/Walters, William 2013: »Actor-Network Theory« and International Relationality: Lost (and Found) in Translation, in: International Political Sociology 7: 3, 332-334.

Beswick, Danielle/Hammerstad, Anne 2013: African Agency in a Changing Security Environment: Sources, Opportunities and Challenges, in: Conflict, Security and Development 13: 5, 471-486.

Bhaskar, Roy 1997: A Realist Theory of Science, London.

Bieler, Andreas/Morton, Adam David 2001: The Gordian Knot of Agency-Structure in International Relations: A Neo-Gramscian Perspective, in: European Journal of International Relations 7: 1, 5-35.

Bigo, Didier 2002: Security and Immigration: Toward a Critique of the Governmentality of Unease, in: Alternatives 27: 1, 63-92. 
Bøås, Morten 2013: Youth Agency in the »Violent Life-Worlds« of Kono District (Sierra Leone), Voinjama (Liberia) and Northern Mali: »Tactics« and Imaginaries, in: Conflict, Security and Development 13: 5, 611-630.

Boltanski, Luc 2012: Love and Justice as Competences. Three Essays on the Sociology of Action, Cambridge.

Bongaerts, Gregor 2007: Soziale Praxis und Verhalten - Überlegungen zum Practice Turn in Social Theory, in: Zeitschrift für Soziologie 36: 4, 246-260.

Bourdieu, Pierre 1977: Outline of a Theory of Practice, Cambridge.

Brown, William 2012: A Question of Agency: Africa in International Politics, in: Third World Quarterly 33: 10, 1889-1908.

Brown, William/Harman, Sophie 2013a: African Agency in International Politics, in: Brown, William/Harman, Sophie (Hrsg.): African Agency in International Politics, Abingdon, $1-15$.

Brown, William/Harman, Sophie (Hrsg.) 2013b: African Agency in International Politics, Abingdon.

Büger, Christian 2013: Actor-Network Theory, Methodology, and International Organization, in: International Political Sociology 7: 3, 338-342.

Büger, Christian 2014: Thinking Assemblages Methodically: Some Rules of Thumb, in: Acuto, Michele/Curtis, Simon (Hrsg.): Reassembling International Theory. Assemblage Thinking and International Relations, New York, NY, 58-66.

Büger, Christian/Gadinger, Frank 2008: Praktisch gedacht! Praxistheoretischer Konstruktivismus in den Internationalen Beziehungen, in: Zeitschrift für Internationale Beziehungen 15: 2, 273-302.

Büger, Christian/Gadinger, Frank 2014: International Practice Theory. New Perspectives, Basingstoke.

Christen, Markus 2010: Eine Neurobiologie der Moral? Einschätzungen der Beiträge von Neurowissenschaften und Verhaltensforschung zum Verständnis moralischer Orientierung, in: Fischer, Johannes/Gruden, Stefan (Hrsg.): Die Struktur der moralischen Orientierung. Interdisziplinäre Perspektiven, Münster, 49-123.

Coole, Diana 2005: Rethinking Agency: A Phenomenological Approach to Embodiment and Agentic Capacities, in: Political Studies 53: 1, 124-142.

Côté-Boucher, Karine/Infantino, Federica/Salter, Mark B. 2014: Border Security as Practice: An Agenda for Research, in: Security Dialogue 45: 3, 195-208.

Côté, Adam 2016: Agents without Agency: Assessing the Role of the Audience in Securitization Theory, in: Security Dialogue 47: 6, 541-558.

Cushman, Fiery 2008: Crime and Punishment: Distinguishing the Roles of Causal and Intentional Analyses in Moral Judgment, in: Cognition 108: 2, 353-380.

De Boeck, Filip/Honwana, Alcinda 2005: Introduction: Children \& Youth in Africa. Agency, Identity \& Place, in: Honwana, Alcinda/de Boeck, Filip (Hrsg.): Makers \& Breakers. Children \& Youth in Postcolonial Africa, Oxford, 1-18.

De Certeau, Michel 1988: Kunst des Handelns, Berlin.

Deleuze, Gilles/Guattari, Felix 1987: A Thousand Plateaus. Capitalism and Schizophrenia, Minneapolis, MN.

Della Porta, Donatella/Diani, Mario 2006: Social Movements: An Introduction, 2. Auflage, Malden, MA.

Dessler, David 1989: What's at Stake in the Agent-Structure Debate?, in: International Organization 43: 3, 441-473.

Doty, Roxanne L. 1997: Aporia: A Critical Exploration of the Agent-Strucutre Problematique in International Relations Theory, in: European Journal of International Relations 3: 3, 365-392.

Emirbayer, Mustafa/Mische, Ann 1998: What is Agency?, in: American Journal of Sociology 103: 4, 962-1023. 
Erskine, Toni 2003a: Assigning Responsibilities to Institutional Moral Agents: The Case of States and »Quasi-States«, in: Erskine, Toni (Hrsg.): Can Institutions Have Responsibilities? Collective Moral Agency and International Relations, Basingstoke, 19-40.

Erskine, Toni (Hrsg.) 2003b: Can Institutions Have Responsibilities? Collective Moral Agency and International Relations, Basingstoke.

Fisher, Jonathan 2013: Structure, Agency and Africa in the International System: Donor Diplomacy and Regional Security Policy in East Africa Since the 1990s, in: Conflict Security and Development 13: 5, 537-567.

Franke, Ulrich/Roos, Ulrich 2010: Actor, Structure, Process: Transcending the State Personhood Debate by Means of a Pragmatist Ontological Model for International Relations Theory, in: Review of International Studies 36: 4, 1057-1077.

Gertel, Jörg/Ouaissa, Rachid/Ganseforth, Sonja 2014: Jugend in der Arabischen Welt, in: Gertel, Jörg/Ouaissa, Rachid (Hrsg.): Jugendbewegungen: Städtischer Widerstand und Umbrüche in der arabischen Welt, Bielefeld, 12-31.

Gibson-Graham, J. K. 2006: A Postcapitalist Politics, Minneapolis, MN.

Gibson, John 2008: The Myth of the Multitude: The Endogenous Demise of Alter-Globalist Politics, in: Global Society 22: 2, 253-275.

Giddens, Anthony 1986: The Constitution of Society. Outline of the Theory of Structuration, Cambridge.

Gill, Stephen 2000: Toward a Postmodern Prince? The Battle in Seattle as a Moment in the New Politics of Globalisation, in: Millennium 29: 1, 131-140.

Griffiths, Ryan D. 2017: Admission to the Sovereignty Club: The Past, Present, and Future of the International Recognition Regime, in: Territory, Politics, Governance 5: 2, 177-189.

Haftel, Yoram Z./Thompson, Alexander 2006: The Independence of International Organizations, in: Journal of Conflict Resolution 50: 2, 253-275.

Hardt, Michael/Negri, Antonio 2004: Multitude: War and Democracy in the Age of Empire, New York, NY.

Hawkins, Darren G./Jacoby, Wade 2006: How Agents Matter, in: Hawkins, Darren G./Lake, David A./Nielson, Daniel L./Tierney, Michael J. (Hrsg.): Delegation and Agency in International Organizations, Cambridge, 199-228.

Hawkins, Darren G./Lake, David A./Nielson, Daniel L./Tierney, Michael J. (Hrsg.) 2006: Delegation and Agency in International Organizations, Cambridge.

Hay, Colin 2002: Political Analysis. A Critical Introduction, Houndmills.

Held, David/Koenig-Archibugi, Mathias 2007: Global Governance and Public Accountability, Malden, MA.

Hellmann, Gunther/Baumann, Rainer/Bösche, Monika/Herborth, Benjamin/Wagner, Wolfgang 2005: De-Europeanization by Default? Germany's EU Policy in Defense and Asylum, in: Foreign Policy Analysis 1: 1, 143-164.

Herborth, Benjamin 2004: Die via media als konstitutionstheoretische Einbahnstraße. Zur Entwicklung des Akteur-Struktur-Problems bei Alexander Wendt, in: Zeitschrift für Internationale Beziehungen 11: 1, 61-87.

Holmqvist, Caroline 2013: Undoing War: War Ontologies and the Materiality of Drone Warfare, in: Millennium 41: 3, 535-552.

Honwana, Alcinda 2006: Child Soldiers in Africa, Philadelphia, PA.

Honwana, Alcinda/De Boeck, Filip (Hrsg.) 2005: Makers \& Breakers. Children \& Youth in Postcolonial Africa, Oxford.

Jackson, Patrick Thaddeus/Nexon, Daniel H. 2013: International Theory in a Post-paradigmatic Era: From Substantive Wagers to Scientific Ontologies, in: European Journal of International Relations 19: 3, 543-565.

Jensen, Michael C./Meckling, William H. 1976: Theory of the Firm: Managerial Behavior, Agency Costs and Ownership Structure, in: Journal of Financial Economics 3: 4, 305-360. 
Koch, Martin 2009: Autonomization of IGOs, in: International Political Sociology 3: 4, 431-448.

Lambach, Daniel 2017: Herrschaft und Agency in der internationalen Hierarchie, in: Daase, Christopher/Deitelhoff, Nicole/Kamis, Ben/Pfister, Jannik/Wallmeier, Philip. (Hrsg.): Herrschaft in den Internationalen Beziehungen, Wiesbaden, 29-50.

Latour, Bruno 2000: Die Hoffnung der Pandora. Untersuchungen zur Wirklichkeit der Wissenschaft, Frankfurt a. M.

Latour, Bruno 2001: Das Parlament der Dinge. Für eine politische Ökologie, Frankfurt a. M.

Latour, Bruno 2005: Reassembling the Social. An Introduction to Actor-Network-Theory, Oxford.

Latour, Bruno 2010: Eine neue Soziologie für eine neue Gesellschaft, Frankfurt a. M.

Leander, Anna 2013: Technological Agency in the Co-constitution of Legal Expertise and the US Drone Program, in: Leiden Journal of International Law 26: 4, 811-831.

Lewis, David/Opoku-Mensah, Paul 2006: Moving Forward Research Agendas on International NGOs: Theory, Agency and Context, in: Journal of International Development 18: 5, 665-675.

Marcus, George E./Saka, Erkan 2006: Assemblage, in: Theory, Culture \& Society 23: 2-3, 101-106.

Mattissek, Annika/Wiertz, Thilo 2014: Materialität und Macht im Spiegel der AssemblageTheorie: Erkundungen am Beispiel der Waldpolitik in Thailand, in: Geographica Helvetica: Schweizerische Zeitschrift für Geographie 69, 157-169.

Mieth, Corinna 2012: Positive Pflichten. Über das Verhältnis von Hilfe und Gerechtigkeit in Bezug auf das Weltarmutsproblem, Berlin.

Müller, Martin 2015: Assemblages and Actor-networks: Rethinking Socio-material Power, Politics and Space, in: Geography Compass 9: 1, 27-41.

Neumann, Iver B. 2002: Returning Practice to the Linguistic Turn: The Case of Diplomacy, in: Millennium 31: 3, 627-651.

Nexon, Daniel H./Pouliot, Vincent 2013: »Things of Networks«: Situating ANT in International Relations, in: International Political Sociology 7: 3, 342-345.

O'Neill, Kate/Balsiger, Jörg/VanDeveer, Stacy 2004: Actors, Norms and Impact: Recent International Cooperation Theory and the Influence of the Agent-Structure Debate, in: Annual Review of Political Science 7: 1, 149-175.

Paharia, Neeru/Kassam, Karim S./Greene, Joshua D./Bazerman, Max H. 2009: Dirty Work, Clean Hands: The Moral Psychology of Indirect Agency, in: Organizational Behavior and Human Decision Processes 109: 2, 134-141.

Podder, Sukanya 2015: The Power In-between: Youth's Subaltern Agency and the Post-conflict Everyday, in: Peacebuilding 3: 1, 36-57.

Pouliot, Vincent/Cornut, Jérémie 2015: Practice Theory and the Study of Diplomacy: A Research Agenda, in: Cooperation and Conflict 50: 3, 297-315.

Reckwitz, Andreas 2002: Toward a Theory of Social Practices. A Development in Culturalist Theorizing, in: European Journal of Social Theory 5: 2, 243-263.

Reckwitz, Andreas 2008: Praktiken und Diskurse: Eine sozialtheoretische und methodologische Relation, in: Kalthoff, Herbert/Hirschauer, Stefan/Lindemann, Gesa (Hrsg.): Theoretische Empirie: Zur Relevanz qualitativer Forschung, Frankfurt a. M., 188-209.

Roff, Heather 2011: A Provisional Duty of Humanitarian Intervention, in: Global Responsibility to Protect 3: 2, 152-171.

Rothe, Delf 2015: Von weitem sieht man besser. Satellitensensoren und andere Akteure der Versicherheitlichung, in: Zeitschrift für Internationale Beziehungen 22: 2, 97-124.

Schäfer, Hilmar 2016: Praxis als Wiederholung: Das Denken der Iterabilität und seine Konsequenzen für die Methodologie praxeologischer Forschung, in: Schäfer, Hilmar (Hrsg.): Praxistheorie: Ein soziologisches Forschungsprogramm, Bielefeld, 137-160.

Schatzki, Theodore 2010: Materiality and Social Life, in: Nature and Culture 5: 2, 123-149. 
Schatzki, Theodore/Cetina, Karin Knorr/von Savigny, Eike 2001: The Practice Turn in Contemporary Theory, London.

Schindler, Sebastian 2014: Man versus State: Contested Agency in the United Nations, in: Millennium 43: 1, 3-23.

Scholl, Christian/Freyberg-Inan, Annette 2013: Hegemony's Dirty Tricks: Explaining Counter-Globalization's Weakness in Times of Neoliberal Crisis, in: Globalizations 10: 4, 619-634.

Schouten, Peer 2014: Security as Controversy: Reassembling Security at Amsterdam Airport, in: Security Dialogue 45: 1, 23-42.

Seckinelgin, Hakan 2006: The Multiple Worlds of NGOs and HIV/AIDS: Rethinking NGOs and Their Agency, in: Journal of International Development 18: 5, 715-727.

Stephen, Matthew D. 2009: Alter-globalism as Counter-hegemony: Evaluating the »Postmodern Prince«, in: Globalizations 6: 4, 483-498.

Stern, Maria/Hellberg, Sofie/Hansson, Stina 2015: Studying the Agency of Being Governed? An Introduction, in: Hansson, Stina/Hellberg, Sofie/Stern, Maria (Hrsg.): Studying the Agency of Being Governed: Methodological Reflections, Abingdon, 1-18.

Tucker, Karen 2014: Participation and Subjectification in Global Governance: NGOs, Acceptable Subjectivities and the WTO, in: Millennium 42: 2, 376-396.

Voelkner, Nadine 2015: Neuer Materialismus in den Internationalen Beziehungen, in: Masala, Carlo/Sauer, Frank (Hrsg.): Handbuch Internationale Beziehungen, Wiesbaden.

Walters, William 2014: Drone Strikes, Dingpolitik and Beyond: Furthering the Debate on Materiality and Security, in: Security Dialogue 45: 2, 101-118.

Weiler, Florian 2012: Determinants of Bargaining Success in the Climate Change Negotiations, in: Climate Policy 12: 5, 552-574.

Wendt, Alexander 1987: The Agent-Structure Problem in International Relations Theory, in: International Organization 41: 3, 335-370.

Wendt, Alexander 1999: Social Theory of International Politics, Cambridge.

Wight, Colin 1999: They Shoot Dead Horses Don’t They? Locating Agency in the AgentStructure Problematique, in: European Journal of International Relations 5: 1, 109-142.

Wight, Colin 2006: Agents, Structures and International Relations: Politics as Ontology, Cambridge.

Williams, David 2013: State Agency and State Formation in Africa, in: Brown, William/ Harman, Sophie (Hrsg.): African Agency in International Politics, Abingdon, 129-142.

Zanotti, Laura 2013: Governmentality, Ontology, Methodology: Re-thinking Political Agency in the Global, in: Alternatives: Global, Local, Political 38: 4, 1-17. 